\title{
L'ARBORETUM
}

\section{DE L'HORT DE DIEU}

PAR

\author{
E.-F. DEBAZAC \\ Ingènicur des Eaux et Forèts \\ $2^{*}$ Section de la Station de Recherches \\ et Expériences forestières - Nancy
}





\section{GÉNÉRALITÉS}

\section{1. - Introduction}

C'est à juste raison que les reboisements du massif de l'Aigoual, dans les Cévennes méridionales, sont bien connus en France et à l'étranger car ils sont très certainement un des exemples les plus typiques d'un remolelage d'un paysage et d'une économie locale sous laction du forestier.

La réussite de cette reforestation, entreprise en application des dispositions des lois de 1860 et de 1882 sur le reboisenent, doit étre attribuéc pour une très large part à la valeur technique et humaine de $G$. FARRE qui fut, de I875 à sa retraite, en qualité d'abord de sous-Inspecteur, puis d'Inspecteur et enfin de Conservateur, l'animateur de cette ature.

Ces rehoisenents, qui ont maintenant atteint lake où toute leur productivité peut étre appréciée, ont été essentiellement réalisés en utilisant des essences résineuses de la flore forestière spontanée française. C"est en effet l'Epicéa (Picca alies Karst $=P$. excelsa Link), le Pin à crochets (Pinus uncinata Ram.), le Sapin (Abics alba Miller) et le Mélèze (Larix decidua Miller) qui furent de beaucoup les plus utilisés. Il faut toutefois mentionner également la place faite dans certaines stations comme la foret domaniale de Saint-Sauveur, au Pin noir d'Autriche (Pinus nigra Arn. var austriaca), qui a été la principale essence utilisée à la mème époque dans les reboisements des Alpes méridionales et de la bordure des Causses. Mais il est intéressant de noter que, tout en menant à bien ces reloisements, G. FABRE pressentit l'intérét que pourraient présenter aussi des essences forestières exotiques, soit d'Europe orientale ou d'Extreme-Orient. soit d'Amérique du Nord. Il faut rappeler en effet que l'introduction d'essences étrangères s'était pourstivie en France surtout deptis la fin du xvm" siècle, à la suite de la prospection botanique de l'Extrème-Orient et de l'Amérique du Nord. Mais ces arbres n'avaient été cultivés, au début, que comme des curiosités botaniques par des 
botanistes collectionneurs. Or, dans les dernières décennies du $\mathrm{x}_{\mathrm{x}} \mathrm{x}^{*}$ siècle, la réussite de certaines d'entre elles laissait entrevoir une utilisation possible en sylviculture. Encore fallait-il expérimenter ces espèces dans des conditions écologiques variées et préciser le domaine de leur emploi. Dans cette voie, G. FABRE fut un novateur, car il proposa, dès 1884 (1) d'asseoir de telles expérimentations dans le massif de l'Aigotual. Ainsi, des plantations d'essences exotiques furent faites à partir de 1885 près de la Maison forestière de SaintSauveur des Pourcils, sur le versant atlantique de 1'Aigoual, entre 900 et $960 \mathrm{~m}$ d'altitude sur des terrains où affleurent des grès et des calcaires, Ces expériences furent continuées par des plantations faites en 1890, à proxinité de la Maison forestière de Puéchagut, sur granite, à $1000 \mathrm{~m}$ d'altitude, sur le versant méditerranéen, puis à partir de 1900 par les plantations de l'arboretum de la Foux, entre 900 et $1020 \mathrm{~m}$ d'altitude, sur schistes, sur versant atlantique [3]. Par la suite, à partir de 1902, cette expérimentation devait s'étendre grâce à la collaboration de Ch. FLAhauct, Professeur à l'Université de Montpellier. C'est alors que furent installés les arboretums de Cazebonne près d'Alzon, à $590 \mathrm{~m}$ d'altitude, sur schistes, sur le versant méditerranéen, de Canayères, à $800 \mathrm{~m}$ d'altitude à la limite des schistes et des calcaires, sur le versant atlantique et enfin ceux de la Fageole, du Trevezel et de l'Hort de Dieu. Ces trois dernières réalisations furent tout spécialement louvre de Ch. Flahavet qui s'était vu confier personnellement, en 1902, la création de jardins botaniques à l'Aigoual par M. le Directeur Général des Faux et Forêts,

Ainsi se trouvaient implantées, vers 1905, un réseau de plantations expérimentales dont il est possible maintenant de tirer de nombreux enseignements.

II est utile de rappeler quels étaient les objectifs de l'installation de ces arboretums et jardins. Ch. Flahault la écrit lui-même à plusieurs reprises: la mission qui lui avait été confiée par l'Administration forestière était " d'organiser à l'Aigoual des observations suivies sur la vie des végétaux dans leurs raports avec le climat et d'élargir le cadre des études physiques entreprises à l'observatoire météorologique (2) en y faisant rentrer les études biologiques si importantes pour la sylviculture (3) $\mathrm{b}$.

En 1904. FtaHAult, dans un rapport présenté au conseil de l'Université de Montpellier [5] fixa les deux buts qu'il se proposait:

1. L'étude spéciale de l'adaptation de certaines essences forestières au climat cévenol.

(1) Un rapport de G. FABRE, dn 11-11-1884 est reproduit par M. NÈGRE [10] p. 94 et 95. 1895 .

(2) L'installation de l'observatoire du sommet de l'Aigoual fut achevée en

(3) Lettre à M. le Directeur de PEcole Nationale des Eaux et Forêts du 31-10-1928. 
2" L'étude générale des végétaux dans leurs rapports avec le climat et en particulier l'étude comparative des végétaux appartenant it des climats considérés comme analogues.

Ce programme de travail fut exposé à nouveau, plus tard, en 1912, an Congrès international de Géographie à Genève, puis en 1913, at Congrès international forestier à Paris.

Enfin, Flahault, lorsqu'il fit un bilan rapide de ces expérimentations, précisa à nouveau ses idées en ces termes (1):

a La végétation spontance est l'expression inmédiate et précise des conditions du nilieu extérieur, du climat et du sol. Les végétaux sont des réactifs d'une extrême sensibilité qui permettront d'apprécier les moindres variations de climat. II est donc particulièrement intéressant d'examiner les rapports de la végétation avec ces variations. Toute tentative d'acclimatation est illusoire. Nous n'acclimatons pas, nous ne réussissons ì introduire un végétal d'un pays dans un autre que sil trouve dans ce pays nouveau pour lui, un ensemble de conditions de climat et de sol identiques a celles qu'il subit dans son pays d'origine ou très peu différent. Il est done très important au point de vue sylvicole de bien connaitre les conditions qui agissent d’une manière si invariable sur une espèce déterminée.

Ce fut là le point de départ, lidée maitresse du progranme que nous avons adopté et dont nous avons poursuivi la réalisation pendant douze ans jusqu'à Theure ou la guerre nous imposa de nouveaux devoirs.

Notre devoir était surtout détudier de façon aussi précise que possible la biologie des especes lignenses spontanées ou introduites, sur leurs linites extrines, sur les conditions de sol, d'exposition, d'association où elles vivent, sur leur végétation plus ou moins active, les dommages quelles subissent de la part du climat, les maladies et dégáts causés par les chanupignons ou les insectes, en un mot les causes limitatives qui agissent sur elles.

Il ne pouvait done plus ètre question d'une place d'expériences; il fallait les multiplier, les étendre en des points favorables pour en coordonner les résultats, les compxurer et en tirer des conclusions pratiques, dont forestiers et agriculteurs puissent bénéficier. "

C'est donc dans le cadre de la mission précitée que Fr.aHault proposa en 1902 la création de trois jardins botaniques, dans des stations où précisénent les tentatives de reloisenent avaient échoué : la première sur les pelouses sèches et les roches du Pic de la Fageole. à $1500 \mathrm{~m}$ d'altitute, à $600 \mathrm{~m}$ d̀ l'Est du sonmet de l'Aigoual (1 $570 \mathrm{~m})$; la deuxième sur les sols tourbeux de la molière du Tré-

(1) Lettre à M. le Directeur de l'Ecole Nationale des Eaux et Foréts du 31-10-1928, 
vezel, à $1330 \mathrm{~m}$ d'altitude, à $500 \mathrm{~m}$ au Sud-( )uest du col de la Serreyrède, et enfin la troisieme dans la combe de l'Hort de Dieu. entre 1250 et $1350 \mathrm{~m}$ d'altitude, à $600 \mathrm{~m}$ au Sud du sommet de l'Aigoual.

Il ne reste que peu de choses des introductions faites au Pic de la Fageole et à la Molicre de Trévezel. Par contre, le $\alpha$ jardin n de l'Hort de Dieu, s'est révélé une expérience extrẻmement féconde. C'est d'ailleurs à l'installation de cette expérience que F LAnaul.T se donna avec une passion toute particulière, de 1903 à 1914. II voulait créer en ce lieu alors sévère, une annexe de l'Institut de Botanique: "Une masure en ruines se trouvait au milieu des terrains de l'Hort de Dieu, ruine respectable car c'est celle de la bergerie ou les botanistes du xvie siecle trouvaient le seul refuge possible sur l'Aigotual (1). „Grâce à différentes subventions, à la générosité d'anis dévoués mais surtout grace à ses propres ressources et à son propre labeur. FLAHAtLT transforma cette bergerie en un chalet-laboratoire où de nombreux étudiants et botanistes purent s'abriter ou séjourner, pendant la belle saison car, du $1^{n+}$ novembre au 15 mai, ce chalet, souvent inaccessible en raison de la neige, restait fermé. Flanatzo y effectuat de longs séjours, sotvent en famille, jusquien 1914. Pendant la guetre de 1914-1918, tous les travaux furent suspendus et, après la fin des hostilités, les difficultés obligèrent Flanacl à renoncer, avec peine, à cette reuvre. En 1929, la gestion de l'Hort de Dieu fut confiée, en même temps que celle des attres arboretums de l'Aigoual, à la Station de Recherches forestières annexéc à l'Foole Nationale des Faux et Foréts, qui continua de nouvelles introductions,

Le programme de FlaHault était extrêmement vaste puisque furent plantées aussi bien des espèces herbacées, des arbustes d'ornement que des espèces légtumières ou fruitières et des essences forestières. Une étude sur le résultat des introductions des plantes herbacées et arbustives du secteur Europe a déjả été publiée |11|. Il importait de donner le résultat des essais des essences forestières,

(1) Lettre à M. le Directeur de l'Ecole Nationale des Eaux et Forèts du 31-10-1928.

\section{C̆.ıсни́: 1.}

La partic centrale de l'Arboretum en août 1910 (cliché FtıHaule). Le clalet laboratoire a été construit. Les plantations, à gauche du cliché, au delà du ravin Pitton de Tournefort, sont celles d'Abies nordmanniana et d'Abies cephalonica. 


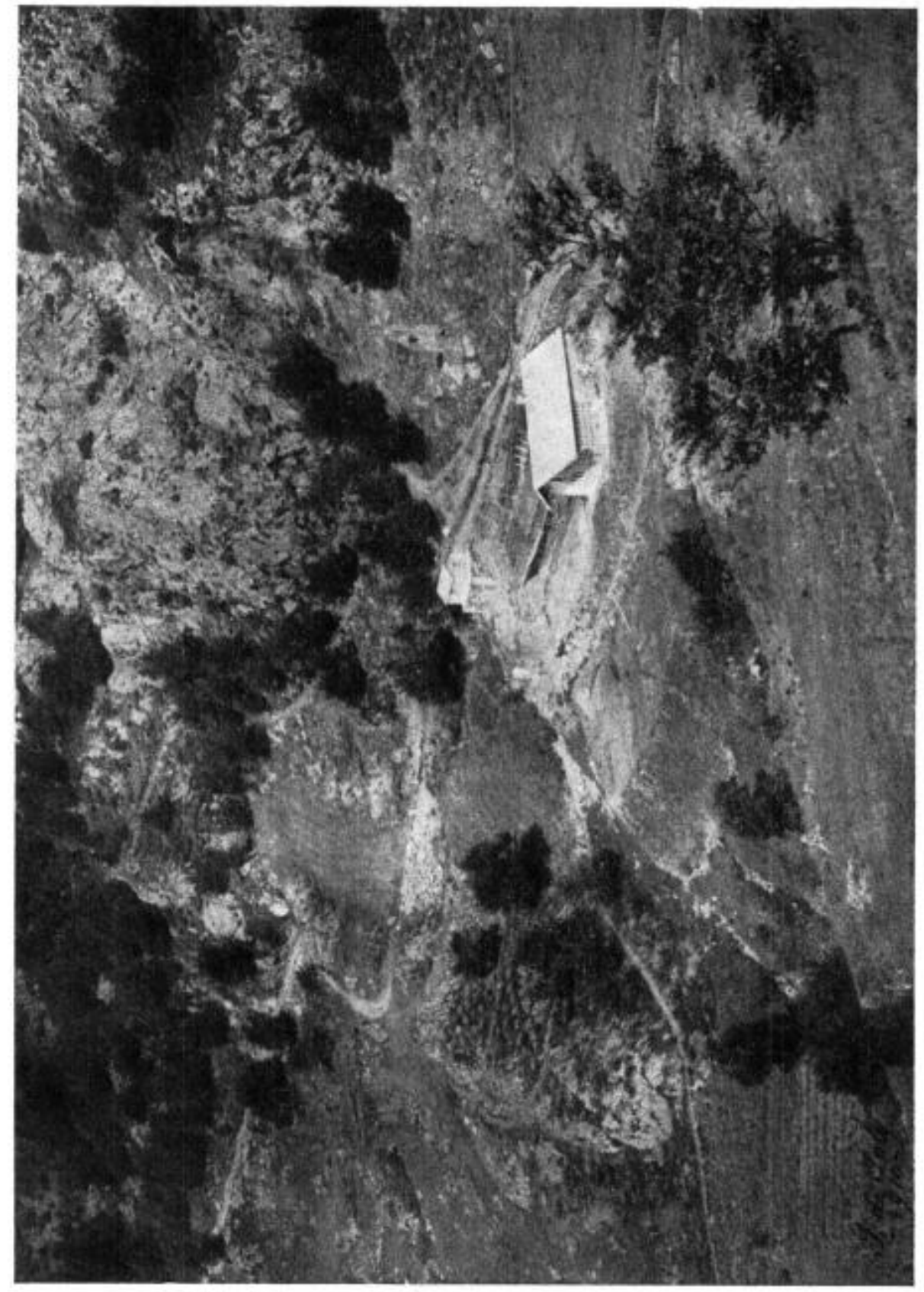




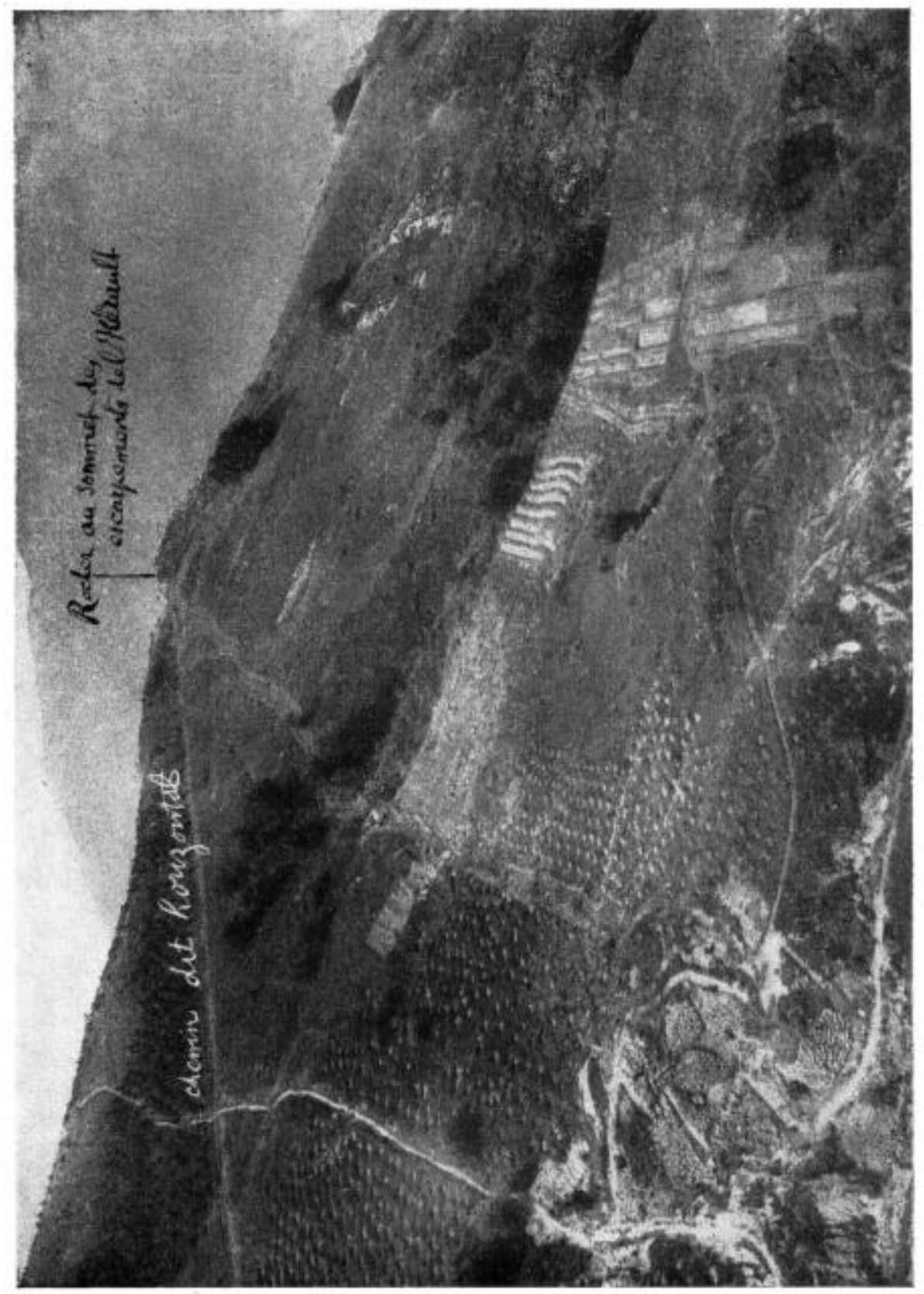


plantées aussi bien par Flaнаutr que, ultérieurement par la Station de Recherches forestières. Tel est le but de ce travail (1).

Les clichés pris par Flahauct ( $n^{\circ} 1$ et 2) (2) dans la combe de l'Hort de Dieu avant et pendant les plantations, permettent d'imaginer comment se présentait le site à cette époque. Autour de la bergerie, construite un peu en dessous des escarpements rocheux, la végétation forestière était réduite à quelques cépées de Hêtre, les roche's étaient presque entièrement dénudées et des pâturages occupaient les parties les moins accidentées au Sud de la maisonnette. Comme il a été dit précédemment, les tentatives de reboisement faites antérieurement avaient échoué. Les seuls plants rescapés étaient quelques pins à crochets et quelques pins sylvestres, ces derniers peut-ètre d'origine « Haguenau ». Enfin, en 1902, vraisemblablement au printemps, des pins sylvestres mentionnés appartenant à la variété rigaensis et des pins laricios (var. calabrica) avaient été plantés. Les premières plantations expérimentales furent effectuées à l'automne 1903, Ces travaux furent laborieux. FLAHAULT rapporte ainsi (3) que " le sol est formé d'éboulis de schistes primaires était si stérile qu'il nous fallu économiser avec avarice tout ce qui pouvait se transformer en terre ou en humus. Au bout de trois ans, nous eûmes ramassé dans des trous du terreau dont chaque arbre planté eut sa ration; les engrais chimiques aussi firent merveille. Grâce à ces soins, le désert de l'Hort de Dieu devient un jardin $n$.

(1) Ch. Franault a laissé de nombreuses notes manuscrites. Certaines ont été adressées en 1928 à M, le Directeur de l'Ecole Nationale des Eaux et Forèts et se trouvent dans les archives de la Station de Recherches; d'autres se trouvent à l'Institut Botanique de Montpellier oủ elles ont pu être consultées gráce à la très grande obligeance de $M$. le Professeur Exrberger. Par ailleurs, différents auteurs ont mentionné les résultats obtenus dans les divers arboretums de l'Aigoual, dont l'Hort de Dieu, notamment Pardé (L.) [12], Nìgre (M.) [10] et RoL (R.) [13]. Enfin, les comptes rendus rédigés par M. le Directeur PoukTEt, chargé de la gestion des arboretums jusqu'en 1958 ont été extrêmement précieux.

(2) Un autre cliché a déjà été publié par Flahauzt [5].

(3) Lettre à M. le Directeur de l'Ecole Nationale des Eaux et Foréts du 31-10-1928.

Les parcelles du Sud-Est de 1'Arboretum (P1. 16, 17, 18, 19, 20, 21, 22 , 23 et 24) le 19 juillet 1909 (Cliché FlahstLt) : on distingue au premier plan a gauche les rocailles du jardin alpin, doù part le chemin $\&$ de lobservatoire », croisant le chemin \& horizontal \$. De chaque cóté du chemin \& de l'observatoire \&, les trous de la plantation de Pinus cembra. Plus à gauche, la préparation de la plantation d'Abies numidica et au-dessous, les planches de la pépinière. Les annotations portées sur le cliché sont de la main de Flahault. 
Les premiers plants furent mis en place en 1903, sans tenir compte de l'origine géographique de l'espèce. Ce n'est qu'en 1904 que Franault adopta une division géographique de l'arboretum en retenant trois compartiments principaux: l'Europe, 1'Asie et l'Amérique (1). Ceci explique certaines anomalies car des plants d'Abies nordmanniana mis en place en 1903 se sont ainsi trouvés dans des parcelles affectées par la suite au compartiment Amérique, alors que des Pseudotsuga menziesii étaient plantés dans les parcelles réservées l'année suivante à l'Europe. Sur le terrain, ces compartiments furent délimités par des ravins auxquels FLAHAuLt donna des noms de botanistes célèbres: Michaux, Thunberg, Pallas, Tournefort, Heller, Desfontaines; certains des sentiers ouverts reçurent également les noms de Rauwolf, de l'Ecluse, Bauhin.

Le premier soin de Flanault fut de créer une pépinière à proximité même du chalet-laboratoire où il put semer et repiquer et assurer ainsi la production des plants qui étaient nécessaires. II semble que la plupart des plants forestiers lui avaient été fournis par l'Administration forestière qui entretenait à l'époque dans le massif de l'Aigoual d'importantes pépinières, notamment à SaintSauveur des Pourcils et à Puéchagut. En ce qui concerne les espéces herbacées et les arbustes, les semis étaient faits at Jardin botanique de Montpellier.

A l'époque, on n'attachait encore que peu d'importance à l'origine des graines. Cependant Flanault a noté souvent les personnes qui lui adressaient les graines et les plants et il est ainsi possible maintenant de connaitre l'origine de quelques introductions. En effet, en plus de l'Administration forestière qui l'autorisait à prélever des plants en pépinière et du Jardin botanique de Montpellier, F LA- $_{\text {- }}$ HAULT recevait des graines de différents correspondants étrangers. Des plants furent en outre achetés chez des pépiniéristes, I.es renseignements connus seront mentionnés dans la $2^{t}$ partie.

Les plantations continuèrent ainsi jusqu'en 1914. FLAIsun.T fit pendant cette période de nombreuses observations et dressa déjả une liste des especes ligneuses cultivées sans succès satisfaisant. II retourna plus rarement à l'Hort de Dieu après la guerre 1914-1918 et nous n'avons eu connaissance que d'observations faites en 1921 et 1925 .

Sous la gestion de la Station de Recherches forestières, des intrnductions ont été reprises entre 1938 et 1941 et. plus récemment. depuis 1961.

\section{II. - Conditions écologiques}

L'arboretum occupe une surface de 20 hat, dans un cirque à l'exposition générale Sud, entre 1250 et $1350 \mathrm{~m}$ d'altitude, stuspendu

(1) Cette division géographique fut adoptée, à la mème époque, dans différents arboretums tel celui d'Amance (M,-et-M.). 


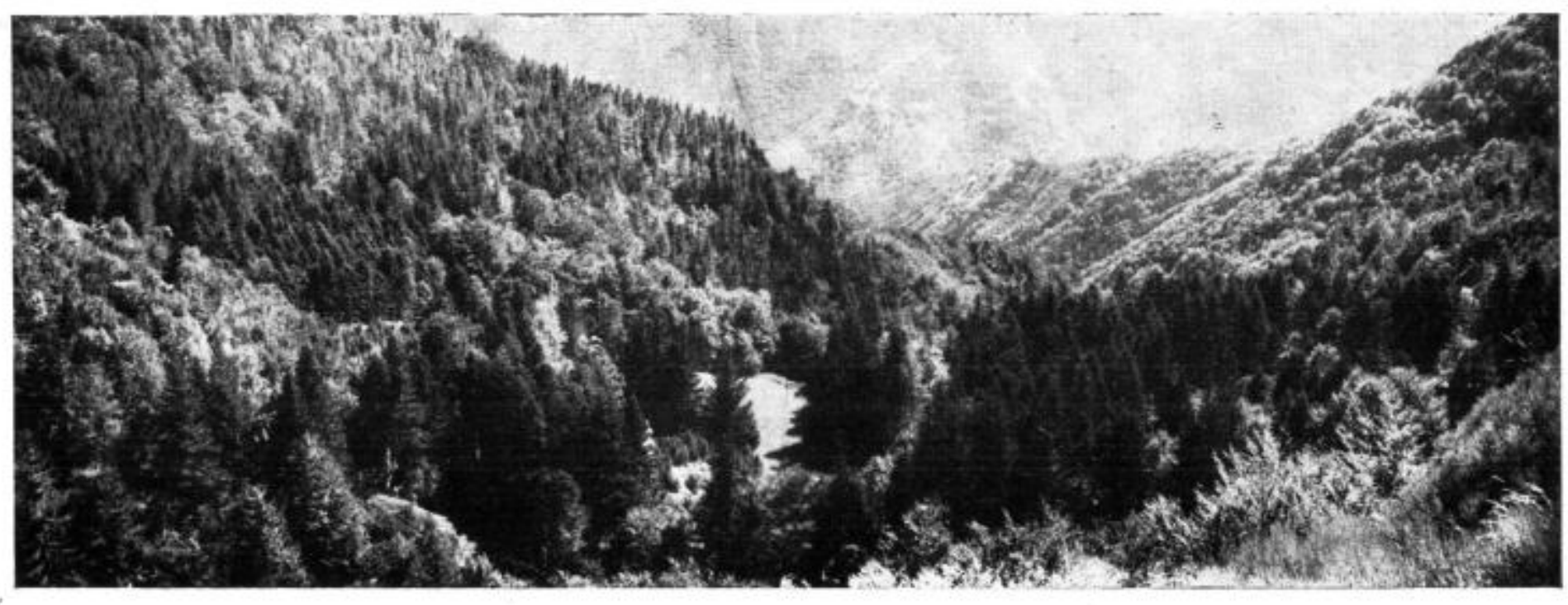

CL.tente 3.

Vue générale de r'Arboretum prise du chemin c horizontal \& (1962). La moitié gauche correspond aux parcelles du cliché 2: le tracé du chemin borizontal se distingue dans le coin gauche en haut. Au premier plan, groupe d'Abies cephalonica. Derrière, placeaux d'Abies numidica et $P$ inus cembra. Au delà, plantation d'épicéas et de pins sylvestres. Dans la moitié droite. at centre, Abies nerdmanniana. Dans le fond, la vallée de l'Héranlit. 
au-dessus de la vallée, sculptée par l'érosion, où l'Hérault prend sá source (1). Le substratum est constitué par des schistes, qui forment les différents contreforts appuyés sur le pilier central granitique du sommet de I'Aigotal. Ces schistes, en raison de lactivité intense de l'érosion sur des pentes fortes en climat à précipitations intenses, ne donnent souvent que des lithosols. Cependant, sur certains replats, notamment sur le versant à l'exposition Sud-Est ainsi qu'au has du versant à l'exposition Ouest, dans des conditions plus favorables à la végétation, là où la couverture forestière était relativement la mieux conservée et où un peuplement de Hêtre en taillis âgé a pu se reconstituer à partir de l'ancien ensouchentent, des sols profonds ont pu se constituer.

Outre les schistes, il faut noter la présence vers la limite NordOuest de l'arboretum, de rochers de granite. Dans les ravins, le mélange de schistes et de granite se retrouve, La présence de ce granite qui n'est pas un élément en place, sexplique par les phénomenes de solifluxion dont l'importance dans la formation des sols du massif de l'Aigoual est grande, conme l'a mis en évidence GaLzin [8].

\section{LE CLIMAT,}

Le massif de l'Aigoual, situé sur la ligne de partage des eaux des bassins des fleuves cótiers méditerranéens et des affluents de la Garonne, se trouve aussi à la limite des influences climatiques méditerranéennes et océaniques. Son altitude donne en outre à son climat un caractère montagnard marqué.

Si aucune observation météorologique n'a été faite dans l'arboretum de l'Hort de Dieu, nous disposons tout au moins des résultats des olsservations faites au sommet mêne de l'Aigoual, à $1554 \mathrm{~m}$ d'altiturle, à $600 \mathrm{~m}$ seulenent à vol d'oiseau du chalet-laboratoire, Des observations sont faites également à Valletaugue, localité située à $378 \mathrm{~m}$ d'altitude, dans la vallée de l'Hératult, au pied du versant sur lequel est accroché l'Hort de Dieu (2).

Les conditions climatiques de latboretum sont certes moins dures, en ratison de la différence d'altitude et de la situation abritée de la station, que celles du sommet de d'Aigotal. Mais tous les renseignements concernant les températures et les précipitations recueillis a l'observatoire sont néanmoins extrêmenent précieux.

(1) $\mathrm{C}$. plan en aunexe et cliché 3 ,

(2) Les renseignements météorologiques sont extraits des publications suivantes:

- Etude climatologique du Bas Languedoc et du Roussillon, Office National météorologique, mars 1942.

- Moyennes mensuelles et annuelles des hauteurs de précipitations en France, période 1901-1930.

Météorologie Nationale, Paris, 1950.

- Normales mensuelles et annuelles des précipitations en France, période 1921-1950, Paris, 1959. 


\section{LA PLEVIOSITÉ.}

Les précipitations sont caractérisées par une hauteur annuelle très forte, avec un maximum d'atutomne très marqué et un minimum d'été. Lat répartition amutelle et mensuelle est précisée dans le tableau suivant :

\begin{tabular}{|c|c|c|c|c|c|c|c|c|c|c|c|c|c|}
\hline & $\mathbf{J}$ & $\mathrm{F}$ & $\mathrm{M}$ & A & M & J & $\mathrm{J}$ & $A$ & S & 0 & $\mathrm{~N}$ & $\mathrm{D}$ & Total \\
\hline Htitude & - & - & - & - & - & - & - & - & - & - & - & - & - \\
\hline $\begin{array}{r}\text { Mont-Aigoual } \\
1554 \mathrm{~m}\end{array}$ & 195 & 150 & 192 & 179 & 190 & 143 & 68 & 110 & 198 & 312 & 246 & 192 & 2175 \\
\hline$(1901-1930)$ & 168 & 155 & 203 & 191 & 211 & 127 & 74 & 83 & 217 & 373 & 302 & 221 & 232.5 \\
\hline (1921-1950) & 131 & 99 & 162 & 181 & 191 & 90 & 71 & 110 & 228 & 280 & 261 & 221 & 2025 \\
\hline $\begin{array}{c}\text { Valleraugue } \\
378 \mathrm{~m} \\
(1901-1930)\end{array}$ & 123 & 131 & 183 & 130 & 130 & 86 & 39 & 60 & 140 & 246 & 206 & 159 & 1633 \\
\hline
\end{tabular}

II est done prohable que la hauteur moyenne des précipitations ì l'Hort de Dieu est de $2000 \mathrm{~mm}$ environ.

Le nombre moyen et la répartition annuelle des jours de pluie au sommet du Mont Aigoual est la suivante:

$$
\begin{array}{ccccccccccccc}
\text { J } & \text { F } & \text { M } & \text { A } & \text { M } & \text { J } & \text { J } & \text { A } & \text { S } & \text { O } & \text { N } & \text { D } & \text { Total } \\
- & - & - & - & - & - & - & - & - & - & - & - & - \\
14 & 13 & 15 & 16 & 15 & 12 & 10 & 9 & 12 & 16 & 16 & 15 & 162
\end{array}
$$

Du mois d'octobre au mois de mai, les précipitations tombent sous forme de neige. T.e nombre moyen des jours avec chute de neige, toujours à l'observatoire de l'Aigoual, se répartit ainsi dans l'année:

$$
\begin{array}{ccccccccccccc}
\text { J } & \text { F } & \text { M } & \text { A } & \text { M } & \text { J } & \text { J } & \text { A } & \text { S } & \text { O } & \text { N } & \text { D } & \text { Total } \\
- & - & - & - & - & - & - & - & - & - & - & - & - \\
7 & 8 & 8 & 8 & 3 & - & - & - & - & 2 & 5 & 7 & 48
\end{array}
$$

Outre les précipitations proprement dites, le brouillard est fréquent aut-dessus de $1000 \mathrm{~m}$ d'altitude, dans l'étage où la hètraic est bien développée. II $y$ a ainsi en moyenne 203 jours de brouillar:l au Mont Aigoual, mais il est vraisemblable que ce nombre doit ètre nettement inférieur à l'Hort de Dieu. 


\section{TFMPÉRATURES.}

Les normales mensuelles des températures maximales et minimales au sommet de l'Aigoual sont données dans le tableau suivant :

\begin{tabular}{|c|c|c|c|c|c|c|}
\hline & J & F & $\mathrm{M}$ & $\mathrm{A}$ & $\mathrm{MI}$ & $\mathrm{J}$ \\
\hline & - & - & - & - & - & - \\
\hline $\max$. & 0.3 & 0,3 & 1,2 & 4,0 & 9.2 & 13,0 \\
\hline min. & $-4,3$ & $-4,5$ & $-3,4$ & $-1,4$ & 3,0 & 6,2 \\
\hline \multirow[t]{3}{*}{ moy. } & $-2,0$ & $-2,1$ & $-1,1$ & 1,3 & 6,1 & 9.6 \\
\hline & $\mathbf{J}$ & A & $\mathrm{S}$ & 0 & $\mathrm{~N}$ & D \\
\hline & - & - & - & - & - & - \\
\hline $\max$. & 16,2 & 16.2 & 12,5 & 8,0 & 3,3 & 1,0 \\
\hline $\min$. & 8,6 & 8.8 & 6.3 & 2,4 & $-1,1$ & $-3,2$ \\
\hline moy. & 12,4 & 12,5 & 9,4 & 5,2 & 1,1 & $-1,1$ \\
\hline
\end{tabular}

Il y a certes, dans ce domaine encore, des conditions moins sévères à l'Hort de Dieu qu'à l'Aigoual, mais il est fort possible que pendant trois mois la moyenne mensuelle soit voisine de $0^{\circ}$. Le nombre de jours de gelée, qui est de 157 at sommet, doit être à l'arboretum probablement supérieur à 100.

\section{LES VENTS.}

Les vents viennent de trois directions nettement dominantes: ce sont le $\mathrm{N}$, le $\mathrm{N}-\mathrm{O}$ et le S. I a fréquence cumulée des vents du $\mathrm{N}$ et du N-O est de $53 \%$, la fréquence des deux directions $\mathrm{N}$ et $\mathrm{N}-\mathrm{O}$ étant du mème ordre de grandeur. Celle des vents du Sud est moindre, sauf en septembre et en octobre. Ce sont ces vents du Sud qui apportent alors les précipitations à caractère torrentiel, d'origine méditerranéenne.

\section{III. - La végétation naturelle}

Dans " la Flore du massif de l'Aigoual " [1], Braun-Blanguet a dressé le catalogue des espéces de la région, depuis la zone de basse altitude jusqu'aux pelouses du sommet. L'Hort de Dieu est très souvent mentionné dans les localités citées. FlaHault a luimème laissé une liste de plus de 200 espèces observées à l'Hort de Dieu. Cependant, un tel répertoire de la flore spontanée de l'arboretum n'ajouterait que peu de choses à la connaissance du site. Il est sans doute d'un plus grand intérêt d'essayer de préciser quels sont les principaux groupements végétaux qui y sont ou y ont été représentés. 


\section{$\stackrel{*}{*}$}

Les quelques taillis vicillis de Hetre prenant par place l'aspect d'une futaie sur souches, permettent d'imaginer quelle a pu ètre la forêt clinacique qui occupait les stations les plus fraiches. Ces peuplements fragmentaires de Hêtre proviennent du développement de cépées disséminées qui subsistaient au moment des premières plantations, comme il a été dit précédemment. La documentation photographique montre combien ces reliques forestières étaient misérables. La seule protection des parcelles, mises à l'abri du pâturage et de la hache pendant un demi-siècle, a done permis la reconstitution de la hetraie là où l'ensouchement était suffisant, à l'Hort de Dieu comme dans la plupart des autres cantons du massif de l'Aigoual. Cette hêtraie montagnarde est très voisine, par sa composition floristique de celle que l'on peut voir, sur des sols plus fertiles, dans la haute vallée du Bonheur, près de la Seyrèrède toujours dans le mème massif. Le Hêtre constitue pratiquement à lui seul la strate arborescente: les autres espèces arborescentes ne jouent qu'un róle plus accessoire. Tel est le cas du Sorbier des Oiseleurs (Sorbus aucuparia). Toutefois, si cette espèce est si abondante dans l'arboretum, cette abondance tient évidemment aux plantations qui en ont été faites près du chalet comme le long des différents sentiers parcourant le compartiment Europe de l'arboretum. Mais grace aux notes de Finhault, il est possible de dire que l'espèce existait déjà spontanément à l'Hort de Dieu.

Dans cette hêtraie, sous la strate élevée du Hêtre, lorsqu'clle est complète, ne se développe aucune strate arbustive. Ia strate herhacée est elle-même peu développée et pauvre en espèces: c'est la Canche flexueuse (Deschampsia flexuosa) qui est souvent la plus ahondante, accompagnée de Poa nemoralis I. et de Holcus mollis I. La Luzule (Luzula nivea DC) n'est jamais aussi fréquente que dans d'autres cantons de hêtraie tels que celui de la Seyrèrède. On trouve également la Verge d'or (Solidago virga-aurca L.), la Raiponce (Phytcuma spicatum) et des Epervières (Hicracium murorum L.). Dans les endroits les plus frais, la Myrtille (Vaccinium myrtillus L.) peut constituer un tapis presque continu. Cet ensemble floristique peut caractériser la hêtraie à moder, qui se distingue nettement des hètraies à mull actif, dont le tapis herbacé est beaucoup plus riche en espèces. Ce dernier type de hêtraie se rencontre pourtant à proximité, par exemple clans le canton de la Dauphine. à l'entrée du chemin de l'Hort de Dieu, à partir de la route de l'Observatoire.

Cet étage montagnard, avec sa hêtraie typique, n'occupe pas cependant la totalité du site, même à l'altitude moyenne de $1.300 \mathrm{~m}$. Lexposition au Sud entraine dans les massifs en limite de la région méditerranéenne, la remontée des éléments de flore des étages inférieurs. L'étage montagnard est ainsi écrasé et entamé d'une part 
par les influences du sommet ef d'autre part par les influences méditerranéennes.

Ainsi, le Chène vert (Qucreus ilca 1..) se rencontre par pieds isolés sur les crétes rocheuses jusqüà $1200 \mathrm{~m}$, mais ne constitue pas à cette altitude, de peuplements ni même de bouquets. Par ailleurs, à l'Hort de Dieu, le Chène pubescent (Qucrcus lanuginosa Lam.) est assez fréquent parmi les cépées de Hêtre. Certains éléments de la chénaie pubescente montent à peu près à cette altitude, tel est le cas de 1'Amélanchier (Amelanclier v'ulgaris Moench) et de l'Erable de Montpellier (.teer monstessulanum L..). L'Erable à feuille d'obier (Acer opulifolium Vill.), assez caractéristique de cette zone disputée entre le Hêtre et le Chêne pubescent, existe dans la parcelle 21. Il y est très vraisemblablement spontané, car son introduction r'est pas notée. Enfin, une espèce est abondante dans ces stations de hêtraie thermophile. II s'agit de l'Alisier blane (Sorbus aria L.) qui est très fréquent. Quelques sujets ont été plantés, mais il était certainement déjà très répandu spontanément. Son abondance, par exemple dans les parcelles 2, 3 et 4, à l'exposition Sud-Fst, permet de penser que le boisement à Alisier blane peut ètre considéré comme une des phases dans la reconstitution progressive de la hetraie thermophile.

Le long des ravins oit l'eau est permanente, c'est la ripisilve à Aune glutineux (Alnus glutinosa Gaertn.) qui occupe le lit du ruisseau et les berges, accompagné de Saules - (Salix cinerea T... S. capraea I.). I.e Tremble (Populus tremula I..) a été noté par Framautr. Ia végétation herbacée qui accompagne ce groupement est caractérisée en outre par la présence de Fougères: Polystichum filix mas. Roth., Athyrim filix femina Roth., Asplenimm trichomanes L.., Polypopodium aulgare...

Si donc, la hêtraie montagnarde à muder et la hêtraic thermophile à Chène pubescent peuvent être considérées comme les deux groupements forestiers climaciques, il n'en demeure pas moins qu'au moment des premières plantations. les terrains de l'Hort de Dieu n'étaient occupés que par des pelouses surpâturées et des landes. Frahault a noté parmi les espèces dominantes la Callune (Calluna v'ulgaris Salisb.), le Genêt purgatif (Genista purgans L.). Genista pilosa L.. Deschampsia flexuosa G., Festuca duriuscula T. et F. ovina L. Ces espèces constituent encore les mêmes groupements, partout où le reboisement n'a pas occupé le sol.

II faut ajouter que dans quelques stations, comme la crète limite Est de l'arboretum, une Fétuque de grande taille. Festuca spadicca L. peut se développer en nappes étendues. Enfin, sur les rochers, s'installe le Genévrier (Juniperus communis L.) auquel se mêle Cotoncaster integerrima Medik. 


\section{CATALOGUE DES ESPECES LIGNEUSES INTRODUITES A L'HORT DE DIEU}

Dans cette deuxième partie sont mentionnées les observations faites à la suite de l'introduction des espèces ligneuses.

Un grand nombre de ces espèces ont été mises en place à l'Hort de Dieu. Beaucoup de ces introductions ont été des échecs qui ont été généralement très rapidement constatés. FLAHAULT a dressé luimême la liste des espèces plantées à l'Hort de Dieu entre 1903 et 1910 " sans succès satisfaisant \#(1).

Ces échecs seront cependant mentionnés diuns le présent catalogue car, en matière d'introduction de végétaux exotiques, ils sont aussi importants que les réussites. II ne faudrait pas toutefois conclure trop hâtivement que la culture de ces espéces est impossible dans les conditions écologiques de l'Hort de Dieu. Cette inadaptation est sans doute probable pour bien des espèces dont les exigences écologiques sont maintenant mieux connues. Mais, en matière de plantations, il $y$ a certes bien d'autres causes d'échec que l'inadaptation écologique! Des essais d'introduction répétés sont nécessaires pour conclure définitivement.

Toutes les introductions faites avec certitude seront portées sur le présent catalogue. FLAHAULt a en effet laissé dans ses notes certaines listes d'espèces qui ne constituaient que des projets de plantations. Beaucoup de ces espèces n'ont pas été réellement mises en place. Seules seront mentionnées les espèces dont la plantation est explicitement notée, par exemple dans le cahier journal tenu pendant les premières années, ou qui ont fait l'objet d'observations ultérieures.

Les espèces sont présentées dans l'ordre des familles adopté par Emberger [4]. A l'intérieur des genres, il a paru plus simple de

(1) Les renseignements provenant de ce document seront mentionnés avec la référence abrégée Fıнниult 1910. 
les classer par ordre alphabétique, sans tenir compte ni des divisions en sections ou sous-sections, ni de la répartition géographique. Dans un index. les principaux synonymes ont été portés.

Pour les essences forestières, il est important de noter le développement des espèces. 11 ne pouvait être question toutefois, en raison du petit nombre de sujets de chaque espece, d'envisager de le traduire par une production en volume. Les circonférences sont celles mesurées à $1,5 \mathrm{~m}$. la hauteur est la hauteur totale sans qu'il soit tenu compte de la hauteur à la première branche ou à l'une des découpes fréquemment utilisées.

Les abréviations conventionnelles suivantes ont été utilisées:

$\mathrm{c}=$ circonférence à $1,5 \mathrm{~m}$. Lorsutue 2 valeurs sont données, il s'agit des valeurs extremes du lot.

$\mathrm{cmx}=$ circonférence à $1,5 \mathrm{~m}$ de l'arbre le plus gros du lot.

$h=$ hauteur totale.

hmx = hauteur de l'arbre le plus haut du lot.

Les mesures ont été effectuées en 1962 sauf indication contraire.

\section{GINKYOACEES}

Ginkyo biloba $\mathrm{I}$.

12 sujets, recus de M. JoLver. Professeur a l'Ecole Nationale des Eaux et Forèts, plantés en novembre 1903. Tous les plants sont morts au cours des deux premières années (I. PARDÉ $\mid 12]$ Fl. 1910).

\section{PINACEES}

\section{Genre Aries}

Abies alba Miller (- A. pectinata D.C.). Sapin pectine.

Cette essence, qui a été largement utilisée dans les reboisements de l'Aigoual. est peu représentée dans l'Hort de Dieu. 11 en existe quelques sujets dans le placeau 23, à coté du jardin alpin. Leur croissance est satisfaisante $(\mathrm{c}=100-130 \mathrm{~cm}$ en 1961).

Abies balsamea (L.) Mill.

Cette espéce, originaire de la région Est de l'Anérique du Nord, a eté assez largement introduite: 151 plants mis en place ì l'automne 1903. - 100 plantés en octobre 1905. Flahault les mentionne comme assez beaux en 1925. NÈGre [10] note Abies fraseri mauvais, mais il s'agit certainement d'Abies balsamea, car rien ne confirme la plantation d'Abies frascri. Le sapin baumier a été ensuite considéré comme disparu de l'arhoretum mais deux sujets ont été retrouvés (Pl. 7), au milieu des épicéas et des mélèzes $(\mathrm{c}=105$ et $58 \mathrm{~cm}-\mathrm{hmx}=10 \mathrm{~m}$ ). Ces sujets sont même fructifères car des semis naturels de 10 à 15 ans se trotvent dans les escarpements rocheux de la parcelle 12, au-dessus du ravin Pallas. 


\section{Abies bormmulleriana Mattf.}

120 plants issus de graines récoltées en Turquic (District de Ayancik - Ornek bólge - Forét de Paza yeri tepesi - alt. : 1100 m) ont été mis en place au printemps 1962 (Pl. 10), à l'automne 1963, il ne restait que 25 plants. I a forte mortalité doit ètre attribuée à la sécheresse de l'été 1962.

\section{Abies cephalonica Loud.}

100 plants d'origine inconnue ont été mis en place en avril 1905. Ce sont vraisemblablement ceux du Pl. 23. Ils ont donné des arbres très vigoureux mais extrêmement branchus.

255 plants ont été plantés à la même date (avril 1905) dans les placeaux 11 et 12 voisins, sous le nom d'Ahies cilicica. Cette espèce de Turquie et de Syrie est bien caractérisée par les bractées du cône incluses, ses aiguilles longues, souples, non piquantes, un peu rabattues vers l'extrénité du rameau. Or, les arbres plantés sous le nom d'A. cilicica n'ont pas ces caractères. La plupart ont des caractères d'Abies cephalonica, avec des aiguilles raides, piquantes, les bractées des cônes très saillantes. Cependant certains, avec des aiguilles un peu rabattues vers l'extrémité du rameatu et des bractées peu saillantes pourraient être des hybrides d'Abies cilicica. II faut ajouter qu'il existe, dans ce même lot, des sujets qui sont incontestablement des Abies nordmanniana et mème un pied d'.Abies mumidica. Tout laisse supposer que les graines dont sont issus ces plants avaient été récoltées dans un arboretum et qu'elles étaient hybridées, et qu'en outre des mélanges ont dù se produire avec d'autres lots, soit en pépinière ou soit au moment de la plantation.

Quoi qu'il en soit, ces arbres ont atteint de fortes dimensions: parmi ceux plantés en alignement le long du placeau 12. les dominants ont des circonférences comprises entre $130 \mathrm{et} 190 \mathrm{~cm}$. I a hauteur maximum est de $22 \mathrm{~m}$.

Parmi ceux plantés dans le placeau 11, certains ont également une bonne croissance. Deux sujets, numérotés C5 et C6 ont été repérés pour leur forme satisfaisante. Leur croissance ces dernières années est la suivante:

\begin{tabular}{|c|c|c|c|}
\hline & \multicolumn{2}{|c|}{ Circonférence } & Accroissement annuel moyen \\
\hline & $1953 \mathrm{P}$ & $1963 \mathrm{~A}$ & sur la circonférence \\
\hline & $120 \mathrm{~cm}$ & $135 \mathrm{~cm}$ & $1.35 \mathrm{~cm}$ \\
\hline C6 & $145 \mathrm{~cm}$ & $173 \mathrm{~cm}$ & $2,54 \mathrm{~cm}$ \\
\hline
\end{tabular}

Tous ces sapins sont vigoureux, très fructiferes. Des semis naturels nombreux se sont installés dans les pelouses voisines, mais ne se développent pas en raison de la proximité des arbres âgés. Cependant. ces arbres sont très branchus, caractère accentué par leur plantation en alignement. Enfin, il faut ajouter que leur valeur fo- 
restière est faible en raison de nombreuses gélivures. Cette sensibilité aux basses températures hivernales a été observée même à altitude moins élevée, ce qui est le cas de larboretum de la Foux $|3|$.

II existe en outre quelques sujets d'Abies cophalonica, dans la parcelle 10 , autour de la stèle dédiée aux botanistes de 1'. Aigoual et à FlanAULt; un de ces stjets a un port multicaule curieux.

Abics concolor (Gord, et Glend.) Lindl.

10 plants ont été mis en place en avril 1905. Fishaust les a mentionnés conme très beaux (1925). C'est également l'appréciation portée par Nìgre en 1930 [10]. II reste 9 sujets d'un comportement irrégulier. Certains sont actuellement dépérissants; d'autres assez vigoureux. Ils sont fructifères ( $\mathrm{hmx}-12 \mathrm{~m}-\mathrm{cmx}=133$ ). Ces arbres constituent un bouquet isolé et des cimes ont été brisées. Quelques semis naturels ont été observés.

Abies grandis (Dougl.) Lindl.

Cette espèce n'a été introduite qu'a l'automne 1963. (Origine des graines: Sisters Ranger Station - Etats-Unis - 45 plants dans le placeau 6).

Abies holophylla Maxim.

40 plants (origine Corée) ont été mis en place sans succès en 1939.

Abies lasiocarpa (Hook.) Nutt. (Abics sulbalpina Fngeln.).

10 sujets ont été plantés au printemps 1939. (Origine des graines: Etat de Washington). I1 reste 4 sujets (hmx $=5 \mathrm{~m}$ ). La fructification a été observée pour la première fois en 1963.

.thies loviana Murr.

25 sujets plantés en avril 1905 ( PI. 2) sous le nom d'Abies lasiocarpa. Ces plants ont été mise en place avec des sujets d'Abies nobilis, Larix leptolepis et Pscudotsuga mensiesii. Cette placette n'ayant pas été parcourue par une éclaircie avant 1961. certains arbres sont morts; d'autres clépérissants ont été enlevés en 1961. II reste (1963) 6 arbres vigoureux dont la croissance est remarquable: le plus gros a $24 \mathrm{~m}$ de hauteur totale et $223 \mathrm{~cm}$ de circonférence (209 en 1959). Ces arbres ont un rhytidome très crevassé et liégeux, ce qui est considéré comme un des caractères distinctifs entre Abies lowiana et Abies concolor. Il faut ajouter que les dendrologues américains (1) comprennent sous le nom d'Abies concolor aussi bien les formes des montagnes rocheuses (Colorado, Arizona, Utah) que celles de Californie et d'Oregon. Or, le rhytidome fortement crevassé et liégeux, outre les autres caractères des aiguilles, parait

(1) Harlow (W.M.) et Harkar (R.J.) Texthook of Dendrology - New York, 1958 - Maul (D.C.) Silvical characteristics of white Fir. California forest and Range Experiment Station, Berkeley, 1958. 


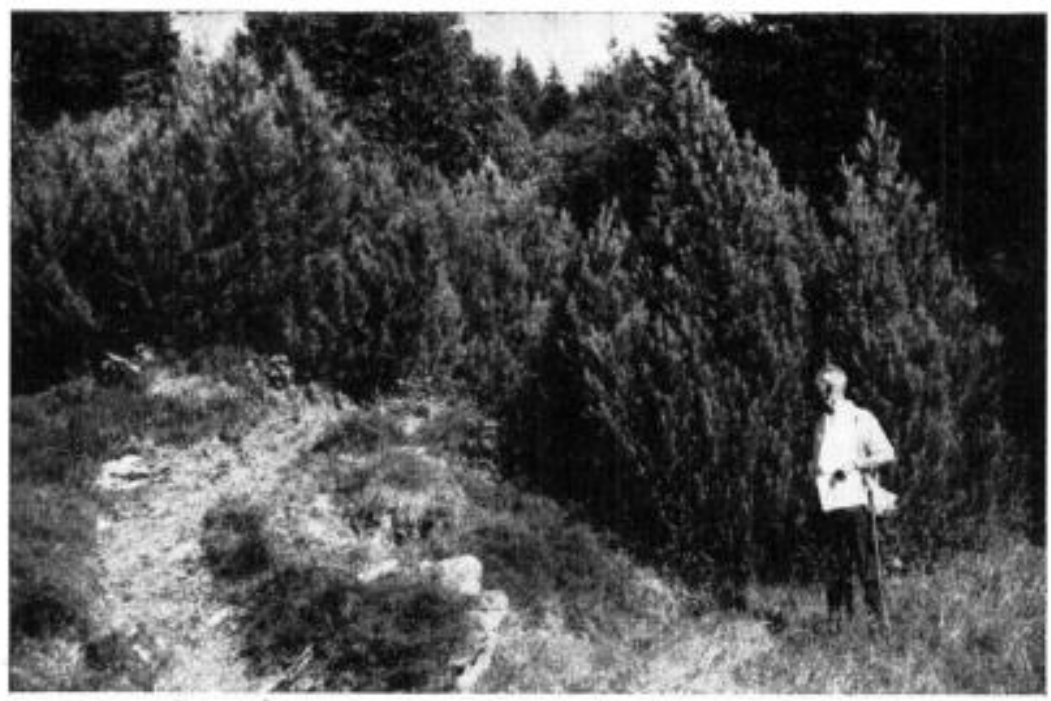

С.генÉ 4.

Plantation de Pimus pumilio (P1. 22) (plantation 1939), en 1962.

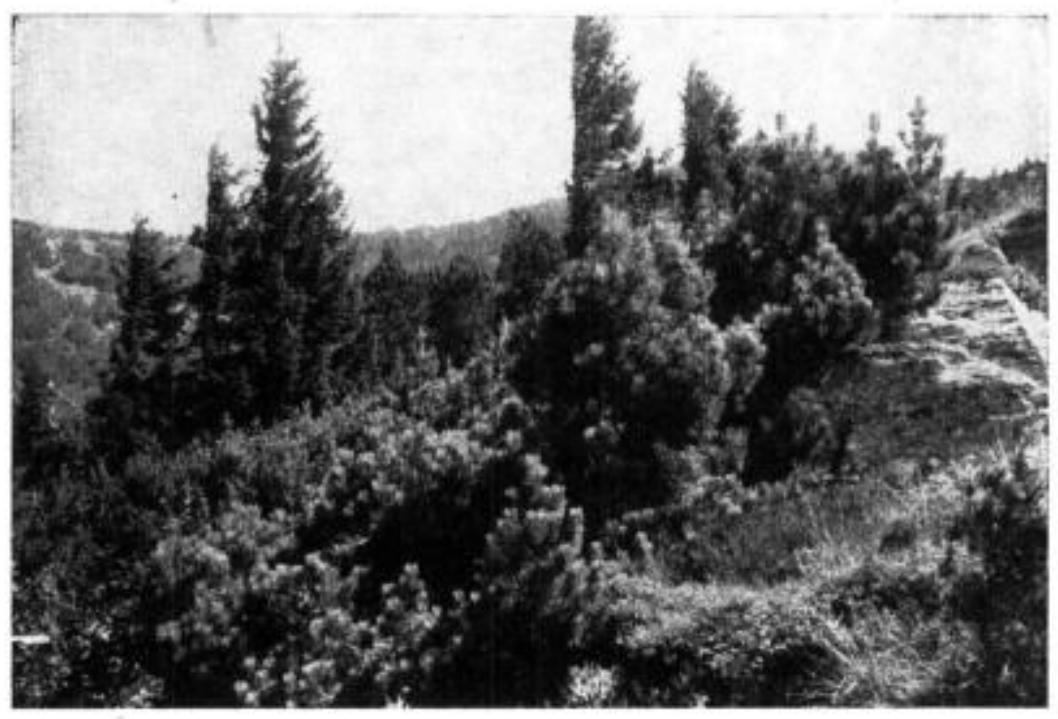

Cliché 5.

Vue du placeau 19, près de la crête constituant la limite Est de l'Arboretum: au premier plan Pinus mughus, ì port couché caractéristique, Derrière, des épicéas déformés e en drapeau $>$. 


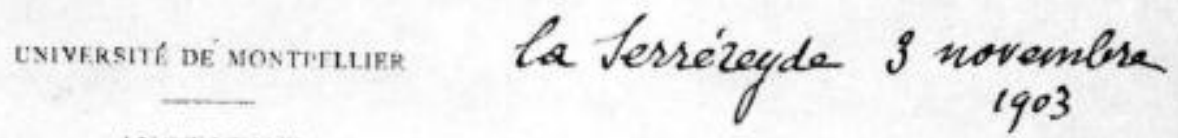

INSTITUT.

Cher momiem en Ami,

of coled be, 12 Ginkgobiloba que vous ave is en la bontede m'arener. In attentant quefen zemercic to'be Aizecteur del'ecole et tho Tiche, fe vour prie de croire ì ma bive gratitude. Hai plantic tout cela hier, malbeureureenent it fout methe fir i ume campagra J'un mois givi a éte active, laborieure of feiconte.

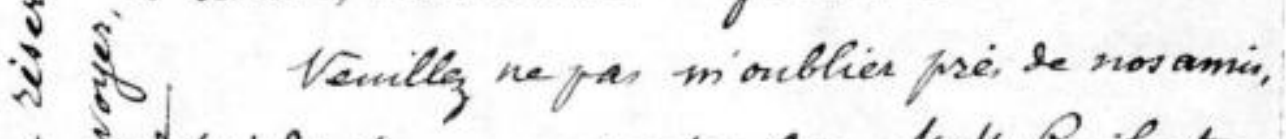

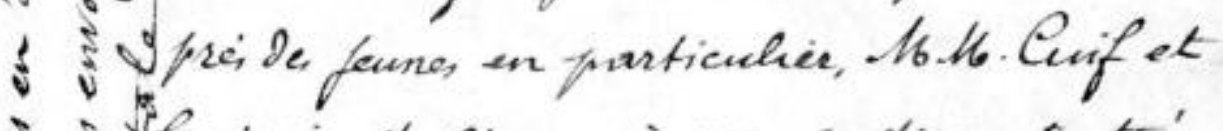

Sinimis el eroyeg a mes sentiments tré.

Os Stananly 
être propre à la forme des chaines cótières. En tout état de cause, les différences morphologiques sont extrêmement nettes entre les sujets existant à l'Arboretum pouvant être rattachés d'une part à Abies concolor sensu stricto et d'autre part à Abies lowiana.

Le développement d'Abies loniana est très nettement supérieur à celui des Abies concolor, mais les qualités de la station sont aussi certainement très différentes.

L'accroissement annuel sur la circonférence du plus gros stjet est de $2,8 \mathrm{~cm}$ depuis 1947.

\section{Abies nordmanniana Spach.}

Cette espèce, dont laire spontanée comprend les étages montagnards du Caucase et la partie Est de la chaine pontique (Turquie) est représentée par plusieurs introductions successives:

Placean 6. Ce sont les arbres plantés en 1903, près du ravin Michaux, dans le compartiment Amérique, avant la division de larboretum en secteurs géographiques $(1) ;$ il reste 8 arbres trìs vigoureux (circonférence de 85 à $142 \mathrm{~cm}$ en 1963) et fructifères.Des semis naturels existent à proximité.

Placeau 10. Il existe un petit peuplement planté en avril 1905 (2) qui est très vigoureux. En 1951, une éclaircie a enlevé une trentaine de tiges. Le peuplement comprend 78 arbres dont la circonférence moyenne est de $80 \mathrm{~cm}(\mathrm{~cm}=132 \mathrm{~cm})$. Ce peuplement est encore très dense. L'élagage artificiel y est très satisfaisant.

11 existe en outre des sujets âgés d'Abies nordmanniana en mélange avec les Sapius plantés sous le nom d'Abies cilicica dans le placeau 11 et daus le placeau 10, autour du monument Flahaut (3). ainsi que dans la partie basse de ce placeat. Tous ces arbres sont vigoureux $(\mathrm{cmx}-175)$ et très fructifères.

Enfin, une nouvelle plantation a été faite au printemps 1963 (P1. 10), Origine des graines: Caucase,

\section{Abies numidica de Lannoy.}

Ce Sapin, dont laire spontanée est restreinte à une station dans les Babors en Algérie, est représenté dans le placeau 12 et dans le placeau 18. Ceux du placeau 12 ont été sins doute plantés en novembre 1903 (4). Ceux du placeau 18 ont été plantés ultérieure-

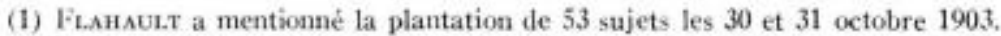

(2) Flahault a mentionné la plantation de 200 sujets du 12 at 15 avril 1905 .

(3) Flahault les note de 1 à $3 \mathrm{~m}$ de haut en 1921.

(4) 71 plants ont été reçus de M. JoLyzr, alors chargé de cours à l'Ecole Nationale des Eaux et Forèts. Ils proviennent done très probablement du lot de graines reçu par FL.icuF en 1897 d'où sont issus également les arbres existant à larboretum d'Amance (M.M.) plantés en 1901 (Cf. cliché $\mathrm{n}^{\circ}$ 6). 
ment, au printemps 1910 (1). Le placeau est dense, Les arbres ont souffert des froids de lhiver 1956, mais ont ensuite bien repris. Certains sujets sont très beaux (cmx $=120)$. Ils fructifient régulièrement. Quelques pieds sont gélivés.

Il existe également des bouquets d'Abies numidica dans le placeat 21 . Un arbre existe également dans le placeau 11 ,

Les résultats donnés par cette espèce sont done intéressants, mais certainement très inférieurs à ceux obtenus avec. Iloies nordmanniana.

Abies pinsapo Boiss.

Cette espece, à aire naturelle isolée dans la sierra de Ronda dans le Sul de l'Fispague, a donné des résultats très médiocres. 215 plants ont èté mis en place à l'automne 1905. II ne reste que quelques sujets (hmx $-7 \mathrm{~m}$ ) peu vigourenx, gélivés.

Abies procera Rehd. (A. nobilis (Dougl.) Lindl.).

$\mathrm{Ce}$ Sapin des chaines cótières de la cóte Pacifique aux EtatsUnis, est représentée dans les placeaux 2 et 6 où il a été planté en 1906 ou 1907 (2). Dans le placean 6, existent 2 sujets isolés (hmx $=18 \mathrm{~m}-\mathrm{c}=160$ et $184 \mathrm{~cm}$ ). Leur croissance est forte (environ $3 \mathrm{~cm}$ par an sur la circonférence depuis 1951) mais des fentes apparaissent sur le tronc. Certaines ont des bourrelets de cicatrisation et pourraient être des gélivures, d'autres sont des fentes de sécheresse. Ces arbres sont très fructifères.

Dans le placeau 2, il restait 7 sujets en 1959, dont 3 secs qui ont ité enlevés. II reste 4 sujets $(\mathrm{cmx}=186 \mathrm{~cm}-\mathrm{h}-23 \mathrm{~m})$. L'accroissement moyen du sujet le plus fort est de $3 \mathrm{~cm}$ par an sur la circonférence depuis 1947, sensiblement égale à celle des plus beaux stijets d'Abies lowiama voisins.

Abies acitchii Lindl.

20 plants (origine des graines: Yatsugatake $L$ fapon) ont été mis en place dans le placeau 9 ou printemps 1939. 11 reste 11 sujets de 6 i $8 \mathrm{~m}$ de haut $(\mathrm{cm} x-60)$. Les deux plus gros ont fructifié pour la prenicere fois en 1963. Ces Sapins ont certainement souffert dans les premières aunées de plantation de ne pas avoir été dégagés. Le comportement de cette espèce des montagnes japonaises est à stuivre. Il faut noter que certaines cimes ont été brisées par le vent.

(1) Flahatzit a mentionné dans ses notes que 400 plants ont ité prélevés en pépinière, le 18 cotobre 1909, pour ètre plantés à l'Hort de Dieu en mars 1910.

(2) D'après les notes de Flahavlt, les plants proviennent de la pépinière de Puéchagut. Il existe encore un exemplaire de cette espèce près de la maison forestière du même nom ( $c=147$ en 1959). 


\section{Genre Pseudotsuga}

Pseudotsuga menziesii (Mirb.) Franco ( $P$. doutglasii Carr $=P$. tarifolia (Poir.) Britton. - Sapin de Douglas.

Cette esssence a été plantée dès les premières années de la création de l'arboretum. 80 plants ont été mis en place à l'automne 1903 dans les parcelles qui devaient ètre affectées par la suite au secteur Anérique et atu secteur Europe. L'origine de ces plants est inconnue. 11 sagit vraisemblablement de plants existants alors dans les pépinières de l'Administration forestière, comme ceux mis en place at la méme époque aux arboretums de La Foux, de Puéchagut et de Cazebonne. Les arbres correspondant à ces premièes plantations semblent ètre ceux des placeaux 2 (Amérique) et 16 (Curope).

Pl. 2, ils appartiennent ì la var. mensiesii Franco (= var. viridis). Finanelt a mentionné quil en restait $40 \mathrm{en} 1921$, de 5 à $10 \mathrm{~m}$ de haut. NÈgre $|10|$ les note a très beaux ” en 1930. RoL [13] estime que cette essence a donné des résultats satisfaisants, mais qu'elle semble souffrir des neiges molles et des vents violents. Effectivenent, ces arbres ont une croissance forte puisque les plus beaux ont $200 \mathrm{~cm}$ de circonférence actuellement. Cependant, beaucoup de cimes sont assées et le peuplement s'en trouve déprécié. Des semis naturels ont été notés (J. Pourter) dès 1951. Ces semis s'installent aussi bien en plein découvert que dans le boisement voisin de Bouleau et d'Alisier blanc, Certains de ces semis ont 8 à $10 \mathrm{~m}$ de haut et commencent eux-mèmes à fructifier.

$P l, 16.3$ sujets de $8 \mathrm{~m}$ de haut existent, au milieu des hêtres et des épicéas près du ravin. Ils sont à rattacher à la var. glauca.

Les plantations de Sapin de Douglas ont été poursuivies en 1904 (127 plants) et à l'automne 1905 (270 plants issus de graines recues de M. A. de VuLaorıs, éduqués à la pépinière de l'Hort de Dieu). Ces plantations semblent correspondre atix arbres existant dans les placeaux 6,7 et 8 . Les caractères des bractées des cónes permettent de rattacher certains à la variété mensiesii, d'autres à la variété glauca, Ils sont d'ailleurs très irréguliers (hmx $=14 \mathrm{~m}$ ), beaucoup ayant souffert de bris de neige.

Il est donc possible de dire que les sapins de Douglas, de provenances inconnues, ont donné à l'Hort de Dieu des résultats inégaux quant i la croissance, certains en sol profond ayant une croissance en diamètre équivalente à celle des Abies procera et Abies lowiana, d'autres se montrant très médiocres, mais tous apparaissant sensibles au vent et à la neige qui sont ici des facteurs décisifs.

\section{Genre Picka}

Picea abies (I..) Karst. (- P. excelsa Link.) - Epicéa.

L'Epicéa, qui a été très largement utilisé dans les reboisements de I'Aigoual, a été planté assez largement dans les différents pla- 
ceaux de l'arboretum, notamment dans les placeaux $7,12,13,14$, 17. 19,21 et 22 (322 plants en avril 1905). L'origine des plants et des graines riest pas connue. Les résultats sont très bons et les irrégularités de croissance doivent ètre attribuées à la différence de qualité de station, I.es arbres des placeaux 17, 19, 22 sont typiquement déformés en "drapeaux " par les vents dominants du Nord; ceux de la parcelle 14 qui, selon Flahault, avaient $6 \mathrm{~m}$ de haut en 1925 , avaient $18 \mathrm{~m}$ en 1959 , au moment oủ certains ont été marqués avant d'être exploités $(\mathrm{cm} x=190 \mathrm{~cm})$. 6 arbres situés près du chalet-laboratoire, le long du ravin Pitton de Tournefort ont de 1.35 a $178 \mathrm{~cm}$ de circonference (hmx $=22 \mathrm{~m}$ ).

L'Epicéa apparait donc comme une des meilleures essences essayées.

Picea ajanensis, ef. Picea hondoensis.

Picea alcockiana Carr.

Flahault a mentionné dans ses notes la mise en place de 100 sujets de cette espéce, en provenance de la pépinière de Puéchagut, en 1907. Il faut rappeler que Picea alcockiand Carr. est synonyme de Picea bicolor Mayr, mais que ce binome a été soument utilisé (Picea alcockiana Veitch) pour des arbres devant ètre rattachés en réalité à l'espèce Picea hondoensis Mayr. Il est possible que ies arbres plantés en 1907 soient cetıx du placeat 11, appartenant à l'espèce $P$. hondocnsis.

Picea asperata Mast.

58 plants de cette espèce chinoise issus de graines récoltées à larboretum des Barres ont été mis en place (Pl. 10) en 1938. II reste 20 sujets dont les plus hauts ont $7 \mathrm{~m}$ et fructifient.

Picea cugcluanni Parry.

15 plants de 5 ans nis en place au printemps 1939 (origine des graines: Etat de Washington). II reste 11 sujets dont certains fructifient abondamment ( $\mathrm{bmx}=8 \mathrm{~m}$ ).

Flanault a mentionné la plantation de 9 Picea engelmami en avril 1905. Il s'agissait probablenent de Picea pungens (cf. P. pungens).

Picea hondoensis Mayr.

Ces épicéas ont été plantés en avril 1905 sous le nom de P. ajanensis. Sur 94 plants il reste 6 sujets $(\mathrm{cmx}=112 \mathrm{~cm}-\mathrm{hmx}$ - $12 \mathrm{~m}$ ) dans les pl, 11 et 12 (Flannult, en 1921, note 9 sujets de $4 \mathrm{~m}$ de haut). Ces arbres à port très étalé, sont très fructifères. Les caractères du rameau (glabre, d'un vert jaunătre la première année, brun-rougeâtre la deuxiène année, le coussinet dépassant peu l'insertion de l'aiguille vers la partie apicale) du bourgeon (conique, très résineux) du cỏne (de 6 à $7 \mathrm{~cm}$ de long. de 1,8 à $2 \mathrm{~cm}$ de large, à bractée en languette acuminée de $5 \mathrm{~mm}$ de long sur $2 \mathrm{~mm}$ de 
large) permettent de rattacher ces arbres à lespece $P$, hondoensis Mayr et non aux especes affines, P. microsperma (Lindl.) Carr. ou $P$. ajancusis Fisher (1).

6 sujets de la même espèce se trouvent dans la parcelle 11. Ils ont le même port mais sont moins développés $(\mathrm{h}=6-7 \mathrm{~m})$. Peutêtre sagit-il des épicéas plantés sous le nom de P. alcockiana en 1907 (cf. P. alcockiana ci-dessus).

Picea omorica (Pancic) Purkine.

10 sujets de cette espéce endémique des montagnes balkaniques, reçu des Pépinières Barbier ont été plantés au printemps 1904 (Pl, 14).

II reste 8 arbres assez vigoureux $(c=58-77 \mathrm{~cm})$, fructifères, nais leur croissunce est nettement inférieure à celle des épicéas communs voisins.

Picea orientalis (L.) Link.

30 sujets existent dans le placeau $9(\mathrm{cmx}-80 \mathrm{~cm}-\mathrm{h}-12 \mathrm{~m})$. Ils sont vigoureux et fructifient, mais leur croissance est faible. Ils ont été mis en place vraisemblablement di l'autonne 1908.

\section{Picea pungens Engelmann.}

Cette espoce a été introduite en 1907 (plants provenant de la pépinière de l'administration forestière de Saint-Sauveur). Elle est actuellement représentée dans le placeau 8 , oi il existe 15 sujets $(\mathrm{hmx}=11 \mathrm{~m})$ et dans le placeau $6(5$ sujets $\mathrm{c}=94 \mathrm{~cm}-\mathrm{h}=9 \mathrm{~m})$, ainsi que par des pieds disséminés dans le placeau 5 . Ces sujets ont eu une croissance assez rapide au début (Flanault a noté une hauteur de 4,50 m en 1925 et Nìgke [10| les mentions " très beaux n) mais ils sont actuellement dépérissants sans avoir atteint des dimensions intéressantes. A la suite des gelées tardives du printemps 1961, il a été noté que les jeunes pousses ont été sérieusement endommagées, alors que les Picca cngelmamni n'étaient pas touchés.

Picea sargentiana Rehd et Wils.

C'est à cette espèce chinoise que semblent pouvoir ètre rapportés 5 épicéas plantés en 1938 sous le nom de $P$. alcockiana, issus de graines récoltées a l'arboretum des Barres. Ils ont de 3 à $4 \mathrm{~m}$ de haut et ont fructifié en 1962.

Picea smithiana Boiss. (= P. morinda I.ink).

10 sujets de cette espéce dont l'aire spontanée est étendue dans l'Himalaya ont été plantés à l'automne 1905. Fitahault a mentionné

(1) Ci. Vassiliev (V.N.).- Epicéas d’Extrëme-Orient de la section omorica Journal botanique de 1'U.R.S.S., tome $35, \mathrm{n}^{\circ} 5,1950$. 
l'échec presque total de cette introduction dès la première année. Des sujets ont cependant subsisté plus longtemps puisque FLAHAUlT indique une hauteur de $1,20 \mathrm{~m}$ en 1921 pour des arbres " périodiquement gelés ". Cette espèce se montre dans d'autres arboretums comme dans celui d'Amance, également sensible aux gelées printanières.

\section{Gienre: Tsuga}

Tsuga mertensiana (Bong,) Carr. ( $-T$, hookeriana (A. Murr.) Carr.

Cette espèce de la région cótic̀re du Pacifique et de l'étage montagnard des chaines cótières est la seule espèce du genre essayée à 1 Hort de Dieu. 11 reste 4 des 5 sujets mis en place dans le placeau 7 en 1904 et 1905. Ces arlores ont entre 4 et $5 \mathrm{~m}$ de haut, mais ils ont été dominés jusqu'en 1961 par des épicéas voisins.

\section{GENRE I.ARIX}

Lari.r decidua Mill ( - I. europea D.C.) - Méléze d'Europe.

Le Mélèze d'Europe, très largement employé dans les reboisements de l'Aigoual, est représenté dans différents placeaux de l'arboretum (7, 12, 16 et 22), où il apparait très vigoureux.

Larix leptolepis (Sieb. et Zuce.) Gord. - Mélèze du Japon.

160 sujets ont été mis en place par erreur daus le compartiment Amérique, Pl. 2 (1905?). Ils y ont donné des résultats excellents. Certains, à proximité des Abies procera et A. lowiana ont été enlevés en éclaircie en 1960 (hmx $-21 \mathrm{~m}-\mathrm{cmx}-145 \mathrm{~cm}$ ).

\section{Ginnre Pseudolarix}

Pscudolarix amabilis (Nels) Rehd.

Filahault mentionne cette espèce dans la liste des espèces ligneuses introduites sans succès (1910).

\section{Genre Centus}

Cedrus atlantica Manetti. - Cèdre de 1 Atlas.

Cette espece introduite au printemps 1905 (53 plants) dans le pliceau 18 et 22 a donné des résultats médiocres. Cependant, les sujets ont parfaitement subsisté jusqu'en $1956(\mathrm{~h}=8-10 \mathrm{~m})$ mais ils étaient moins beatux que les Cèdres du Liban mentionnés ciaprès. Ils niont pas résisté aux basses températures de février 1956. Il ne reste plus que deux sujets peu vigoureux $(\mathrm{h}=4 \mathrm{~m})$ à proximité du jardin alpin. Il faut ajouter que cette essence a parfaitement supporté les froids de 1956 à l'arboretum de La Foux 
à $900 \mathrm{~m}$ d'altitude, mais a souffert à l'arboretum de Saint-Sauveur. à $1000 \mathrm{~m}$ d'altitude. L'altitucle maximale d'utilisation pratique peut donc ètre fixée à $900 \mathrm{~m}$ dans la région de l'Aigoual.

Cedrus deodara (Roxb.) Loud.

Plantés par Finhault à l'automme 1903 (32 plants). 90 sujets ont été plantés à nouveau à l'automne 1905, mais en 1910 FLAHAULT mentionnait que les arbres étaient à peu près tous morts et les derniers survivants ont été notés buissonnants en 1921. Nìgra: |10| les note "mauvais " en 1930 ; ils ont par la suite totalement dispart.

Cedrus libani l.oud. - Cèdre du Liban.

Plantés à l'automne 1905 dans le placeau 9, ces arbres ont en une croissance médiocre dans les premières années. D'après les notes de Flanault, ils avaient de 1 à $3 \mathrm{~m}$ en 1921. A l'entrée de l'hiver $1955-1956$, il restait 65 sujets $(c=40-130 \mathrm{~cm})$. Aucun de ces arbres n'a résisté aux froids de lhiver 1956.

\section{Genke Pines}

Pinus banksiana Lamb. - Pin de Banks - Pin gris (Canada).

Il apparait, d'après les notes de Flahault, que cette espèce. dont laire naturelle est étendue dans le Nord-Est de l'Amérique du Norcl, a été introduite à 2 reprises: une première fois à l'automne 1905, avec des plants recus de 7.EDERBAuER à Mariabrunn (Autriche), la deuxième fois, entre 1906 et 1908 , avec des plants provenant de la pépinière de l'administration forestière à Puéchagut. Le comportement de cette espèce était déjà noté " très mauvais " par Nìgre en 1930. Il ne reste actuellement qu'un seul sujet. peu vigoureux, couché.

Une nouvelle introduction a été faite à l'automne 1963 dans le placeat 6 (origine des graines : chute à Murdock, Canada).

\section{Pinus cembra L. - Pin cembro - Arole.}

Ce Pin, spontané dans l'étage montagnard supérieur et l'étage subalpin des montagnes de l'Europe moyenne, a été essayé dès 1905 car FLAHAult mentionne alors la plantation de 53 plants. Il n'est pas possible de préciser ce que sont devenus ces plants, car une nouvelle plantation a été faite en 1907 dans les placeaux 16 et 17 , de part et d'autre du sentier de l'observatoire. Dans cette dernière plantation, le Pin cembro a fort bien rétsssi. II constitue un peuplement dense dont la hauteur moyenne est de $10 \mathrm{~m}$. Les sujets les plus gros ont $0,70 \mathrm{~m}$ de circonférence. Il faut noter que la plantation a été faite en touffes et n'a pas été suivie des éclaircies néces- 
saires, si bien que les croissances des différents pieds sont très inégales. Ces arbres fructifient.

Pinus contorta Douglas - Lodgepole Pine.

Cette essence, dont laire naturelle est étendue dans la région Ouest de l'Amérique du Nord, n'était pas représentée jusqu'à présent dans l'arboretum. Trois provenances ont été mises en place à lautomne 1963 (origine des graines: Athin. Smithers et Kamlonp district. Canarla). It faut rappelet qu'elle est très utilisée en GrandeBretagne pour rehoiser les stations dans les conditions de sol et de climat les plus difficiles, notamment dans des sols tourbeux en climat maritime froid.

Pinus griffithii Me Clelland $(=P$. excelsa Wall. $)$ - Pin de I'Himalaya.

106 plants mis en place à lautomne 1950 sont tous morts très rapidement. Aucun nouvel essai de cette importante essence de l'Himalaya n'a été effectué.

Pinus leucodermis (Ant.) Markraf.

60 plants de ce pin, dont l'aire naturelle est réduite et morcelée dans les montagnes du Sud-Est de l'Europe, ont été plantés à l'automne 1905. Ces plants avaient été reçus de Zederkaukr à Mariabrunn (Autriche). Cette introduction a bien réussi. Les arbres constituent un bouquet dans le placeau 13, au-dessus du sentier Rauwolf, malgré la quualité médiocre de la station. Cette espèce se montre donc très bien adaptée à la dureté du climat, mais la croissance est faible: la hauteur maximale est de $12 \mathrm{~m}$. Trois sujets, numérotés parmi les plus gros ont $0,92 \mathrm{~m}, 0.68 \mathrm{~m}$ et $0.88 \mathrm{~m}$ de circonférence. De plus, la décroissance des troncs est particulièrement forte. II n'en demeure pas moins que cette essence offre des possibilités intéressantes pour le reboisement des stations dans des conditions climatiques rudes ou. de toutes les façons, une forte production ligneuse ne peut pas ètre attendue.

\section{Pinus mughus Scop. - Pin rampant.}

Ce Pin est la forme oric tale du P'in de montagne, spontanée dans les Alpes centrales et orientales ainsi que dans les chaines balkaniques, 50 plants, adressés par ZEDER BAUER à Mariabrum (Autriche) ont été mis en place à l'automne 1905. Ce sont sans doute ceux du placeau 17. En outre, en 1911, des plants de 2 ans ont été reçus du Service de la Recherche forestière d'Autriche à Mariabrum. Ce sont sans doute ceux existant sur la créte limite Est de l'arboretum (placeatux 19, 20 et 21). Tous les sujets de cette espèce existant à l'Hort de Dieu sont donc vraisemblablement issus de graines récoltées dans les Alpes autrichiennes. 1ls ont le port couché caractéristique de l'espèce et constituent une brousse compacte de $2 \mathrm{~m}$ de haut, assurant une excellente cotverture du sol, mais totalement dépourvue d'intérêt pour la production ligneuse. 
Pimus nigra Arnold.

Des introductions des différentes sous-espèces géographiques de cette espèce collective ont été effectuées.

s.sp. Laricio Poiret - Pin Laricio de Corse.

Des plantations de la variété calabrica, effectuées probablement au printemps 1902, done avant la création de l'arboretum, sont mentionées par Flaнavi.t. II n'est pas possible de préciser ce que sont devenues ces introductions.

De nouvelles plantations ont été faites à l'automne 1903 dans une parcelle ultérieurement affectée à la flore américaine (P1. 4). Le peuplement est assez bien venant: ceprendant, près de la crète à la limite Ouest de larloretum, dans une station battue par les vents, les cimes sont déformées en drapeaux ou brisées $(\mathrm{cmx}=150 \mathrm{~cm}-$ $h m x=15 \mathrm{~m}$ ).

110 sujets mentionnés appartenant à la var.corsicana ont été plantés à l'automne 1905 dans le compartiment Furope. Il ne semble rester aucun survivant.

ssp. nigricans - Pin Noir d'Autriche.

Il existe des sujets appartenant à la var. austriaca, disséminés clans le placeat 2, introduits en même temps que les Pins à crochets voisins. Ces arbres sont mérliocres $(\mathrm{cmx}=100-\mathrm{hmx}=10$ m).

\section{Pinus peuce Grisb.}

Cette espèce, dont laire spontanée est morcelée dans les montagnes des Balkans, n'est représentée que par 2 sujets (P1. 1.3). sur le sentier Rauwolf ( $\mathrm{c}=67 \mathrm{et} 69 \mathrm{~cm}-\mathrm{h}=9 \mathrm{~m}$ ). Ces plants ont été reçus des pépinières Calmphtout et plantés au printemps 1904. Leur croissance est lente. mais il faut noter que le sol est superficiel à cet endroit-là.

De nouvelles introductions ont été faites à l'automne 1963 (origine des graines: Igrichte, Bulgarie) dans le Pl. 10.

Pinus ponderosa $\mathrm{I}$ aws.

Ce pin américain, à aire spontanée vaste dans les Montagnes Rocheuses et dans les chaines cótières de la région pacifique, n'est plus représenté que par 4 sujets. PI. $6(\mathrm{hmx}=8 \mathrm{~m}-\mathrm{cm}=75 \mathrm{~cm})$. 73 plants reçus des pépinières Barbier, avaient été mis en place au printemps 1905, dans une station un peu mouilleuse. En 1925. il ne restait déjà plus que 6 sujets de 1 à $2.50 \mathrm{~m}$ de haut (FLAHAult). II faut ajouter qu'à l'arboretum de Cazebonne, dans le massif de r'Aigoual, près d'Alzon, à $590 \mathrm{~m}$ d'altitude, existent 2 beaux sujets $(\mathrm{en} 1959 \mathrm{c}=125$ et $205 \mathrm{~cm}$ ). 
Pinus resinosa Ait.

Des plants de cet espèce du Nord-F-t de l'Amérique du Nord ont été mis en place à l'automne 1963 (origine cles graines: Saint-Philomène, Canada).

Pinus pumilio Haenke.

600 plants de ce Pin quui est une des formes du Pin de montagne, dont laire naturelle se trouve dans les Alpes Centrales, les Sudétes, la Forêt noire, ont été plantés dans le placeau 18, au printemps 1938. La réussite a cté satisfaisante (hn $-3-4 \mathrm{~m}$ ).

Pintes rigida Mill. - Pitch Pine.

Des plants de ce Pin de la région centre-Est des Etats-Unis ont été mis en place dès lautomne 1903 (50 plants) puis à nouveau au printemps 1905 (100 plants fournis par les Pépinières Barbier). II ne reste plus qu'un setul stjet très peu vigoureux dans le placeau 5 .

Cette espèce est également représentée à larboretum de Cazebonne où, malgré des conditions écologiques beatucoup plus favorables, elle n'a donné que des résultats très médiocres.

Pints strobus I. - Pin Wevmouth. - White Pine.

133 plants de ce Pin qui est tue importante espèce de l'Fst de TAmérique du Nord. ont été mis en place en avril 1905. D'autres sujets, éduqués à la pépinière de l'administration forestière de Puéchagut, ont été plantés ultérieurement, sans doute au printemps 1907. Dès 1925, selon les notes de FLAHault, il ne restait plus que 7 sujets (placeatu 6). Nìgre $|10|$ les mentionne a assez vigoureux $n$. Il ne reste actuellement que 9 arbres, au total, dans le placean $6(\mathrm{~cm} x=1,40 \mathrm{~m}-\mathrm{hmx}=10 \mathrm{~m})$ et quelques sujets dans le placeat1 2.

Cette espèce montre manifestement une très mauvaise résistance au vent. qui est à l'Hort de Dieu un facteur important: toutes les cimes ont été brisées à plusieurs reprises. Son comportement est done très différent de celui de Pimes petre, espèce pourtant très voisine, mais de tempérement montagnard: une des différences entre les 2 espèces, outre les caractères des cònes, est précisément le caractere de la cime. Alors que chez Pinus strobus, la cime est irrégulière et ouverte, chez Pims pouce, elle est régulière et dense, ce qui explique la meilleure résistance de cette dernière essence at vent.

\section{Pinus sylvestris $\mathrm{L}$.}

Cette importante essence, dont laire est étendue dans les étages montagnards à travers l'Eurasie, est spontanée sur les versants atlantiques du massif de 1'Aigoual. Il en existe ainsi des peuplements naturels de belle forme dans les forêts domaniales de SaintSauveur, de Lanuéjols (Gard) et de Meyrueis (Lozère). 
Le Pin sylvestre a en outre été largement utilisé dans les reboisements, souvent à défaut de Pin à crochets, comme l'explique Nígre [10]. Ce sont des graines récoltées en Auvergne qui ont été probablement utilisées alors. Il apparait que toutes les introductions de cette provenance faites par semis, à haute altitude, ont été des échecs. Cependant, l'origine "Auvergne " n'est pas certaine et il est possible que parmi les graines utilisées, certaines tout au moins, aient été récoltées dans la région d'Haguenau (Bas-Rhin (NÈGre [10, p. 52]). Dans la périmètre de l'Hort de Dieu, Flanault mentionne la présence, au moment de l'installation de l'arboretum, de quelques pieds de Pin sylvestre de Haguenau, "brisés par les neiges n. 11 existait également de jeunes plantations, datant probablement du printemps 1902, de Pin sylvestre de Riga. Ce sont sans doute les pins sylvestres des placeaux 3 et 4 , mis en place en même temps que les pins laricios. Enfin, Flahault (1925) a mentionné des Pins sylvestres d'Auvergne qui existaient au-dessus du chalet laboratoire.

Au moment des premières plantations de l'arboretum, des pins sylvestres de Riga ont été mis en place en mélange avec des pins laricio dans le secteur Europe, à l'automne 1903. De nouvelles plantations de cette mème origine ont été effectuées à l'automne 1905 (105 plants). En fait, des plantations plus importantes ont été faites puisque les placenux 17, 18 et 19, sur le versant orienté au SudEst, sont occupés par des plantations de Pin sylvestre, dont les caracteres de forme permettent de penser qu'il s'agit bien de la race de Riga. Ces arbres ont dû être très beaux au début puisque Négre les mentionne comme tels $|10|$. En fait, le comportement de ces arbres est décevant, car si la rectitude du füt est excellente, la croissance est médiocre: la circonférence moyenne est de $0,50 \mathrm{~m}$ environ et la hatuteur moyenne de $10 \mathrm{~m}$. En outre, dans les stations certes très exposées au vent, des arbres se montrent extrêmement fragiles dès qu'ils ont atteint une certaine hauteur et très inférieurs aux épicéas qui se trouvent à proximité. De nombreuses tiges sont cassées à mi-hauteur et l'avenir des arbres encore sur pied apparait tris incertain.

\section{Pinus uncinata Ramond - Pin à crochets.}

Ce Pin, qui est la forme occidentale du Pin de Montagne et dont les boisements naturels sont situés dans l'étage montagnard supérieur et l'étage subalpin dans les $A$ pes occidentales et surtout dans les Pyrénées, a été très largement utilisé dans les reboisements de l'Aigoual. II a été introduit soit par semis soit par plantation en touffe, et il a permis de reboiser tous les terrains nus en altitude. II faut noter cependant que cette espèce ne se montre pas longévive et que dans les reboisements qui ont dépassé $50-60$ ans, de nombreux dépérissements se produisent. Elle remplit toutefois parfaite- 
ment le ròle d'essence de premier hoisenent, avec une production ligneuse intéressante.

Le Pin à crochets est représenté dans l'arboretum quoiqu'il n'ait pas été planté dans un but expérimental. En effet, son utilisation était déjà entrée dans la pratique forestière courante au moment de l'installation de l'arboretum. Cependant, il en existe notamment dans les placeaux 1 et 2 où de nombreux sujets commencent d'ailleurs à sécher sur pied.

\section{ARAUCARTACEFS}

Araucaria araucana, K. Koch $=A$, imbricata Pav,

10 sujets ont été plantés en octobre 1907. Ces plants se sont maintenus, quoique peu vigoureux ( $h m x=2,50$ ) à T'abri des hétres (pl. 3) puis sont morts à la suite des froids de l'hiver 1956. Il faut préciser que cette espèce a résisté aux froids de ce méme hiver â Parboretum de la foux |3|.

\section{TAXODIACEES}

Sequeit sempervirens (D. Don) Endl.

4 sujets ont été plantés par Fl.aнnul.t en 1904. Ces plants étaient tous morts dès la $3^{*}$ année (Finhanlt, 1910).

\section{Sequoiadendron giganteum (I.indl.) Buchholz.}

44 plants ont été mis en place le 31-10-1903 dans les placettes qui devaient etre affectées par la suite aux compartiments Europe et Amérique. II n'en reste que dans les placeaux 6 et 7 . Beaucoup étaient morts dans les premières années et les vivants étaient a misérables " (Flahault, 1910). Cependant, les survivants étaient mentionnés comme très beaux par Finhault en 1925. Il reste actuellement 6 sujets qui ont atteint une taille notable $(\mathrm{cmx}=220 \mathrm{~cm})$.

Tarodium distichum (L.) Rich - Bald cypress - Cypres chauve.

Cette espèce de la plaine cótière du Sud-Est des vallées du bassin du Mississipi a été essayée, 90 plants ont été mis en place à l'automne 1905. L. PARDÉ [12] les mentionne presque tous morts en 1908, Un peu plus tard, Flahault les note tous morts (1910).

\section{CUPRESSACEES}

Biota orientalis Endl.

Un seul sujet de cette espèce, à aire étendue en Asie orientale, a été planté, sans succès, à l'automne 1905 .

Calocedrus decurrens (Torrey). Florin - Heyderia decurrens (Torr. Roch.) $=$ Libocedrus decurrens Torr.

Cette espèce de Californie n'est mentionnée par Fl.ahault que sur la liste des espèces cultivées sans succès, avec l'observation \& disparu dès la $2^{*}$ année 3. 
Chamaecyparis lawsoniana (A, Murr) Parl.

La date de plantation de cette essence, dont l'aire naturelle est dans l'Ouest de l'Amérique du Nord, n'est pas connue, Elle est représentée par une ligne de sujets dans le placeau 6. Finhault les mentionne en 1921, de 2 a $3 \mathrm{~m}$ de haut. Le comportement de cette espéce est assez satisfaisant puisque ces arbres ont actuellement de 10 à $12 \mathrm{~m}$ de haut.

\section{Juniferus sabina L.}

Une plantation de 25 sujets faite à l'automme 1905, n’a pas réussi.

Juniperus virginiana L. - Eastern red Cedar.

Une introduction de 3 plants a été faite en octobre 1903. Ces plants sont mentionnés comme misérables par Flahault (1910). Ils n’ont pas été retrouvés par la suite.

Thuja occidentalis L. - Northern white cedar.

34 plants de cette espèce, dont laire naturelle est étendue dans l'Est de l'Amérique du Nord, ont été mis en place à l'automne 1905. Flanault (1910) la mentionne comme " misérable ». Il ne reste que 3 sujets (placeau 6) dont le plus grand a $3 \mathrm{~m}$ de haut.

Thuja plicata Donn. - Western red Cedar.

Une première introduction de 100 plants de cette essence forestière de l'Ouest de l'Amérique du Nord a été faite à l'automne 1905. Cette plantation a été un échec total.

Une nouvelle introduction a été faite en 1938 et 1939 (17 plants). 11 ne reste qu'un seul sujet (placeau 6) de $4 \mathrm{~m}$ de haut.

Thujopsis dolobrata Sieb. et Zucc.

48 plants de cette espèce, qui n'a qu'un intérêt ornemental, ont été plantés au printemps 1905. PAkDÉ, en 1908 [12] mentionne qu'il n'en reste que quelquesuns, Flahault les note \& misérables \$ (1910). Ils n'ont plus été mentionnés par la suite.

\section{TAXACEES}

Taxus baccata L. - If.

Des pieds d'If ont été plantés à l'automne 1905, puis en 1907. Il en reste dans le compartiment Europe, de moyenne vigueur.

\section{BETULACEES}

\section{Genre Alnus}

Alnus incana (L.) Moench. - Aune blanc.

Espèce plantée à l'automne 1905 (103 plants). Flle s'est parfaitement installée dans les ravins et des cépées vigoureuses se trouvent dans les ravins Thunberg. Michaux et Heller. 
Aluss viridis D.C. - Aune vert.

Cette espèce, dont l'aire naturelle est limitée en France, à l'étage subalpin dans les Alpes, a été introduite en 1909 (30 plants recus de M. Kathriver à Sarnen, Underwald. Suisse), Il en existe quelques cépées assez vigoureuses près du chalet lahoratoire, le long du ravin Heller.

\section{Genke. Betula}

Betula alleghaniensis Britton ( $=B$. hutea Michx.).

Ce Bouleau de l'Fst américain a été introduit sans succès en 1905 (11 plants issus de graines adressées par Vilmorin-Andrieux en janvier 1903).

Betula lenta L.

Espèce de l'Fst de TAmérique du Nord, introduite sans stoccès (57 plants en 1905).

Betula pubescens Ehrh.

Betula verrucosa Erhrh.

Les deux especes de Bouleau de notre flore ont été assez largement utilisées dans les reboisements de 1'Aigoual. C'est en général la deuxième espéce qui est de beaucoup la plus fréquente. Elle est d'ailleurs spontanée sur le versant atlantique du massif de l'Aigoual, mais les bouleaux existants dans le périmètre de 1'Arboretum doivent être considérés comme introduits. FlaHAUlt mentionne des plantations a lautomne 1905 (5 plants) puis en 1907 (100 plants provenant de la pépiniere de l'Administration forestière à Puéchagut),

\section{FAGACÉES}

\section{Genre Fagus}

Fagus grandifolia Ehrh. ( $=F$, americana L.).

Cette espèce yui est, dans la région florale de l'Est américain, l'homologue de notre Hêtre, a été introduite sans succès (50 plants, éduqués au Jardin Botanique de Montpellier, mis en place ì l'automne 1905).

\section{Genke Quercus}

Quercus ilicifolia Wangenh.

Une introduction de ce Chẻne de l'Est américain a été faite sans succès (61 plants éduqués à la pépinière de l'Hort de Dieu, mis en place à l'automne 1905). Ces plants étaient mentionnés comme misérables par FLAHAULT (1910). Ils se sont tout de méme maintenus longtemps (RoL. [13]).

Quercus macrocarpa Michx.

Cette espéce d'Amérique du Nord a été introduite $(6$ plants mis en place à l'automne 1905). Elle était déjà notée comme " misérable " par Flahault (1910). Il ne reste que 2 sujets de $2 \mathrm{~m}$ de haut (placeau 6). 
Quercus petraca Liebl. (= Q. sessiliflora Salisb.) - Chène sessile.

Un semis par potet a été fait en octobre 1903 dans le placean 5 , près du chemin horizontal ( 26 potets et 25 glands par potet). Cette introduction a donné un bouquet de Chêne sessile, actuellenent à l'état de haut perchis de 12 a $13 \mathrm{~m}$ de haut. Le port de ces arbres, à trone assez rectiligne, se distingue nettement des chẻnes spontanés qui doivent plutót ètre rapportés à lespèce Quercus pubscens Willd., mème lorsque les caractères de pubescence du rameau d'un an et de la feuille sont très atténtiés.

Quercus robur L. (Q. pedunculata Ehrh.) - Chène pédonculé.

Des sujets de Chêne de faible dimension dans le placeau 6 , présentent les caractères de cette espèce. Leur origine est inconnue.

Quercus ruhra $\mathrm{L}$.

190 plants de cette espèce américaine ont été mis en place, san- succès, à l'automne 1905 .

\section{CORYLACEES}

GenRe Corylus

\section{Corylus avellana I. - Coudrier - Noisetier.}

Le Coudrier est commun sur le versant atlantique du massif de 1'Aigoual. II est plus rare sur le versant méditerranéen. II n'est pats mentionné dans la liste des espèces spontanées dressées par Fi.AHAULT. Les sujets qui existent dans larboretum doivent done ètre considérés comme introduits. Une plantation de 4 pieds est mentionnée en avril 1905 .

Corylus colurna L.

Cette espece, dont laire spontanée se trouve dans la péninsule balkaniunue, est mentionnée seulement sur la liste des espèces cultivées sans suecès (F FAHAULT, 1910).

\section{Genre Carpinus}

Carpines carolimiana Walt. (C. americana Michx.).

Le Charme d'Amérique a été introduit en 1905 (46 plants). Une plantation ultérieure aurait été faite en 1906 ou 1907. Cette espèce, notée encore en 1921 par Fi.AHAUL, semble avoir disparu.

Carpinus betulus L. - Charme.

Le Charme est représenté par quelques sujets dans les placeaux 16 et 18 (plantations de lautomne 1905 et de 1907). Cette essence ne donne que des sujets très médiocres.

\section{Genre Ostrya}

Ostrya virginiana (Mill.) K. Koch.

2 sujets de cette espèce d'Amérique du Nord ont été plantés à l'automne 1903. Ils sont notés comme morts e après 9 ans s sur la liste de Flahault (1910). 


\section{SALICACÉES}

Populus simonii Carr.

Genre Porulus

Ce Peuplier, originaire du Nord de la Chine, a été introduit en 1904 (24 plants). FLahault (1910) le note misérable, et NÈgre [10] médiocre.

Populus tremula L.

Cette espèce est notée comme spontané à l'Hort de Dieu (BraunBlangUet), mais une plantation a été faite en outre à l'automne 1905.

\section{Genre Salix}

Les espéces suivantes ont été retrouvées dans le jardin Alpin (Nozeran [11]): Salix pyrenaica Gouan (planté en 1907). S. caesia Vill., S. capraca L., S. grandifolia Q. (planté à l'automne 1908). S. purpurea L.

Clmus americana L.

\section{U.MACEES \\ Genre Ulmus}

Cet Orme de l'Est de l'Amérique du Nord a été introduit à l'automne 1905 (76 plants) dans le placeau 6, à proximité du ravin Thunberg: il s'est parfaitement maintenu et constitue un houquet dont les arbres les plus hauts ont $8 \mathrm{~m}$.

Ulmus glabra Huds. $(=U$. monlana With $=U$. scabra Miller $)$. Orme de montagne.

Cette espece est spontanée sur le versant atlantique du massif de l'Aigoual; quelques plants ont été plantés en 1904.

\section{POLYGONACEES}

Genre Polygonum

Les espèces asiatiques, à grandes feuilles à limbe cordé, souvent subspontanées en France, sont représentées par un massif à proximité du monument et également dans une corbeille des arbustes asiatiques où Polygonum sacchalinense est extrèmement vigoureux.

Celtis occidentalis $\mathrm{L}$.

\section{Genre Celtis}

Cette espèce n'est représentée que par' un seul sujet planté en 1907 (PI. 6). de $1 \mathrm{~m}$ de haut seulement.

Celtis caucasica Willd. et $C$. sinensis Pers, ont été plantés sans succès (F $F_{\text {LA- }}$ HAULT, 1910). 


\section{GenRe ZeLKova}

Zelknza serrata (Thumb) Mak. (Z. acunimata Planch.).

La plantation de 71 sujets de cette espèce asiatique, effectuce à lautomne 1905 , a totalement échoué.

\section{MORACEES}

Broussonetia pafyrifera (L.) Vent.

Cette espèce originaire d'Extrême-Orient est mentionnée par Fl.sHatLt sur la liste des espèces cultivées sans succès (1910).

Juglans cinerea $\mathrm{L}$.

JUGLANDACÉES

Genre Juglans

Un sujet de ce Noyer américain, planté à l'automne 1905, s'est maintenu * misérablement.

Juglans nigra L.

Cette espèce américaine est mentionnée sur la liste des espèces cultivées sans succès (FLAнAULT, 1910).

\section{Genre Pterocarya}

Pterocarya stenoptera D.C.

Espèce mentionnée sur la liste des espèces cultivées sans succès (F LaHaul.t $1910)$.

\section{Genre Carya}

Carya illonecnsis (Wangenh.) K. Koch. $(=C$, olizaeformis Michx.).

3 sujets de Pacanier ont été plantés, sans succès, en 1907 (Ftrallscl. 1910).

\section{RUTACEEES}

\section{Genre Zanthoxylum}

Des sujets de Zanthoxylum americanum Mill. et de Z. piperetum (L.). D.C. ont été introduits, sans succès (Frahaurt, 1910), (plantations de l'automne 1905 ).

\section{SIMARUBACEES}

Ailauthus altissima (Mill.) Swingle (A. glandulosa Desf.).

Espèce introduite sans succès (F1.лнкUL., 1910).

\section{ANACARDIACEEES}

\section{Genre: Cotinus}

Cotinus coggygria Scop. (Rhus cotinus L.).

Cette espèce est rare dans les Cévennes (Braun-Blangurt) [1]. 10 plants ont été mis en place à l'automne 1905, notés par la suite \& misérables ? par FLAHAULT (1910). Ils semblent avoir fotalement disparu. 


\section{ACERACEES}

Acer campestre L.

\section{Genre Acer}

Cette espèce, très commune sur les terrains calcaires de causses qui bordent le massif de l'Aigoual au Nord-Ouest, est assez rare dans le massif granitique et schisteux. 4 sujets plantés ì l'automne 1905. Un, très peu vigoureux, a subsisté, dominé par le bouçuct d'épricéa du placeau 14.

Acer insigne Boiss.

Essence mentionnée par FLAHAULT (1910) sur la liste des espèces cultivées sans succès.

Acer macrophyllum Pursh.

C'est à cette espéce de l'Ouest américain que semble devoir ètre rapporté un Erable de $3 \mathrm{~m}$ de haut, ne portant pas de fructifications, existant dans une des corbeilles darbustes asiatiques du placeaı 10, où il aurait done été mis en place par erreur.

Acer neguedo L.

Espèce mentionnée par Fiннлец (1910) sur la liste des espèces cultivees sans succès.

Acer pensylianicum L.

Espéce mentionnée par Franater (1910) sur la liste des espèces cultivées sans succès.

Acer platanoides L. - Erable plane.

Espèce spontanée mais rare dans le massif de l'Aigoual. Elle figure sur une liste d'espèces plantées en 1907.

Acer pseudoplatanus L. - Erable sycomore.

Cette espèce, qui ne doit pas être considérée comme spontanée dans le massif de 1'Aigoual (Braun-Blanguet |1|), est cependant très souvent subspontanée. Elle a été introduite dans l'arboretum (15 sujets en avril 1905) où elle est très vigoureuse dans les stations fraiches.

Acer saccharinum L.

Espèce mentionnée par Frahaula (1910) sur la liste des espèces cultivées sans succès.

\section{HIPPOCASTANACEES}

Différentes espèces du genre Aesculus ont été introxluites: A. californica (Spach.) Nutt, A. parviflora Walt (= A, macrestachya Michx.) ainsi que A. $x$ carnea Hayne ( $=$ A. rabicunda Loisel) ont été plantées sans succès (FLAнасLт, 1910).

Il reste des survivants peu vigoureux, près du chalet laboratoire d'Aesculus hippocastanum L., dont 37 plants ont été mis en place à l'automne 1905 puis en avril 1907. 


\section{CORNACEES}

Aucuba japonica Thumb.

\section{Gienre Aucuba}

11 plants ont été mis en place à l'automne 1905, sans succès.

Cornux stolonifera Michx.

\section{Genkf Cornus}

Cette espèce d'Amérique du Nord, dont 10 pieds ont été mis en place à lautomne 1905, s'est vigoureusement installée le long du ravin Michaux, dans le placeau 6 , où elle a tendance à devenir envahissante.

Abelia uniflora R. Brown.

\section{CAPRIFOI.IACERS}

Genre Abetia

Espèce introduite en avril 1907 et mentionnée par FLaHaur (1910) sur ta liste des espèces cultivées sans succès.

\section{Genke Viburnum}

Viburnum pubescens Pursh. (=V. venosum Brit.).

Cette espèce, introduite sans doute en 1907, s'est maintenue dans le placeat 6 , à proximité des sujets d'Abies procera.

Viburnum lantana L. et $V$. opulus L. ont été introduits sans succès (FLAначL, 1910).

\section{Genre Weigelia}

Weigelia florida (Sicb. et Zuce.) A.DC. (= Diervillea florida Sieb. et Zucc.).

Cet arbuste ornemental, originaire d'Extréme-Orient, introduit au printemps 1907, s'est maintenu dans une des corbeilles créées pour les arbustes asiatiques (placeau 10).

\section{GENRE SyMPHORICARpos}

Symphoricarpos albus Blake.

Cet arbuste d'Amérique du Nord, très souvent cultivé pour lornement, introduit en 1905, a bien rétssí et a tendance à devenir envahissant.

\section{GenRe Lonicera}

Plusieurs espèces de ce genre, aussi bien américaines ( $I$. sempervirens L., L. ledebourii Eschsch. L. prolifera Rehd. (= L. sullizantii Gray)), quasiatiques (L. orientalis Lamb. (= L. kesselringii Reg.), L. japonica Thunb. (= L. brachypoda DC.)) ont été plantées avec des plants éduqués au Jardin botanique de Montpellier. Toutes ces espèces semblent avoir disparu. Seul, un sujet de L. iberica Bieb. var microphylla Dip, de l'Iran, a été retrouvé dans une des corbeilles d'arbustes asiatiques (placeau 10). II faut noter en outre la présence dans le jardin alpin de Lomicera alpigena $\mathrm{L}$. et de L. pyrenaica L. [11].

\section{CEI.ASTRACEES}

Evonymus latifolia Scop.

\section{Genre Evonymus}

Introduit, sans succès, Flahault (1910), 


\section{AQUIFOT.IACEFS}

llex aquifolitm L. - Houx.

Cette espèce est spontance dans les ravins frais du massif de l'Aigotal. Quelques pieds ont été en outre plantés, sans doute en 1907.

\section{RHAMNACÉES}

Parmi les espéces du genre Rhamanus, les espèces suivantes ont étét introduites: Rhamaus alpina $\mathrm{L}$. (planté en 1907) dont il reste des pieds vigoureux dans le jardin alpin, $R h$. frangula (planté en 1907) dont des pieds sans dotte subspontanés se trouvent le long du chemin de l'observatoire et $R h$. pallasii Fish, et Mey., d'Asie Mineure, qui semble avoir disport.

\section{OLEACEES}

\section{Gente Fraxinus}

Fraxinus excolsior $\mathrm{L}$. - Frène,

Cette especce est spontanée le long des ravins dans le massif de 1'Aigoual, Il en existe en particulier dans le ravin de larboretum. Cette espèce n'a cependant pas été mentionnée par FLAHAcLT dans la liste des especes de la flore de l'Hort de Dieu et il est probable que les frènes existants sont ceux plantés à l'automne 1905 (55 sujets).

Différentes espèces de frènes, américaines (Fraxinus anericana L. (-F, alba Marsh.), $F$. pensylvanica Marsh. ( $=F$, lanceolata Borkh.), $F$. pubescens, $F$. oregona Nutt.) soit asiatiques $(F$, mandshurica Rupr.) ont été introduites en 1905. Seul, l'échec de l'introduction de F. mandshurica a été mentionné par Flahauli (1910), qui a simplement noté le peu de vigueur des Fraxinus alba et $F$. oregona. II reste actuellement dans le placeau 6 différents sujets de vigueurs diverses, peu fructifères, dont les caractères permettent de les rapporter à Frarinus pensyleanica Marsh., certains pieds i la forme pubescente (F. pubescens Lam.) "Red Ash), la plupart à la forme glabre "Green $\Lambda \mathrm{sh}$ ". Les arbres les plus hauts ont 7 a $8 \mathrm{~m}$.

\section{Genre Forsytita}

Forsythia viridissima Lindl.

Cette espèce, originaire de Chine, très cultivée pour l'ornement en raison de sa floraison très précoce précédant la foliaison, a été introduite à lautomne 1905. Elle est mentionnée sur la liste des espèces cultivées sans succè́s satisfaisant (FLAHAUL.T, 1910), ainsi qu'me autre espéce du méme genre, ègalement asiatique, $F$, suspensa Vahl.

\section{Genre Jasminum}

Jasminum nudifloram Lindl.

L'introduction de cette espèce (printemps 1907), originaire de Chine et souvent cultivée pour l'ornement, n'a pas réussi (FlaHaUl, 1910). 


\section{Genre Ligustrum}

Différentes espèces asiatiques, provenant des semis effectués par FinnaulT au Jardin botanique de Montpellier, ont été plantées en 1907 (L. compactum Hook et Thomas $=$ L. yunnanense L. Henry), L. sinense L. var statentonii (DC) Rehd., L. ibota Sieb, et Zuce, L. quihoui Carr, $L$. amurense Carr.). Les échecs de ces introductions ont été rapidement constatées (Fínhaut, 1910).

\section{Genre Syringa}

Les espèces suivantes ont été introduites: Syringa vulgaris L. du Sud-Est de l'Europe (1905), $S$. persica L. (1905) d'Asie et $S$. pekinensis Rupr. (= Ligustrina pekinensis Regel) (1907).

\section{TILIACEES}

Tilia platyphyllos Scop. (- T. grandifolia Ehrh.).

Le Tilleul à grandes feuilles est spontané mais rare dans le massif de l'Aigoual. 7 sujets ont été plantés à l'automne 1907, près du ravin Pallas où certains pieds se montrent vigoureux.

\section{BERBERIDACEES}

\section{Genre Mahonia}

Mahonia aquifoliam (Pursh) Nutt.

Cette espèce américaine, très souvent cultivée pour lornement, a été introduite sans strcès en 1907 (FLABAUL,T, 1910).

\section{Genre Berberis}

Berberis amurensis Rupr.

La présence de Berberis amurensis Rupr. est mentionnée par Flahault sur la liste des espèces cultivées sans succès satifaisant (1910). Un plant vigoureux, pouvant ètre rapporté à cette espèce, proche de B. valgaris L existe dans la corbeille supérieure d'Asie (placeau 10).

\section{ERICACEES}

\section{Genke RHODODENDRON}

Les deux espèces de la flore française, $R$. ferrugineum $L$. et $R$. hirsulum, plantés à lautomne 1905 dans le jardin alpin, s'y sont parfaitement maintenues.

\section{Rhododendron ponticum $\mathrm{L}$.}

Cette espèce, dont l'aire naturelle est disjointe, en Asie Mineure d'une part et dans le Sud-Ouest de la péninsule ibérique, a été plantée à plusieurs reprises (75 sujets reçus des Pépinières Bakmik au printemps 1904, 100 à lautomne 1905 et sans doute encore en 1907). Fille a remarquablement réussi et constitue un beau massif à proximité de la stèle.

Rhododendron smirnovii Trautv.

Cette espèce, dont l'introduction n’a pas été mentionnée par FL.AHault, caractérisée par un tomentum blanchátre dense sur les jeunes rameaux et à la face inférieure des feuilles, se montre très vigoureuse. Elle existe dans une des corbeilles d'arbustes asiatiques du placeau 10 . 


\section{HAMAMELIDACEES}

\section{Genre Pakrotia}

Parrotia persica C.A. Mey.

Espèce notée par Flahault sur la liste des espèces n'ayant pas été cultívées avec succès (1910).

\section{Genre Parkotiopsis}

Parrotiopsis jacquemontiana,

Espéce notée par Fl.aHault sur la liste des espèces cultivées sans succès satisfaisant (1910).

\section{Genre Liquidambar}

Liquidambar styracifha $\mathrm{L}$.

90 sujets de cette espèce américaine, plantés à lautomne 1905. If s'étaient maintenus jusquen 1910 puisque Flahallt a mentionné alors qu'ils étaient très peu vigoureux et qu'ils n'aoútaient pas, mais n'ont pas été retrouvés par la suite.

\section{SAXIFRAGACEES}

Un sujet, qui pourrait ètre rapporté à Détzia sieboldiana Max. existe dans une des corbeilles du compartiment Asie. Il s'agit peut-être des sujets introduits sous le nom de Deatsia staminea $\mathrm{R}$. Br. II existe également des pieds de Philadelphas non déterminés.

\section{ROSACEES \\ Genke Physucarpus}

Physocarpus opulifolius (L.) Maxim.

Espéce existant dans une des corbeilles d'arbustes asiatiques, dont l'introduction n'a pas été mentionnée par Flahavlr.

\section{Genre Spiraea}

Spiraca salicifolia L.

Cette espèce, intraduite dans le compartiment Amérique par erreur, puisque son aire est eurasiatique, s'y est répandue en nappe, par drageonnement.

\section{Genke Holodiscus}

Holodiscus discolor (Pursh.) Maxim.

Cette espéce s'est également répandue dans le placeau 6 .

\section{Genke Cotoneaster}

Deux espéces de Cotoneaster ont été plantées en 1907 par Flahault dans le placeau 10, dans le compartiment Asie: $C e$ sont $C$, multiflora Bge $(=C$. reflexa Carr.) et $C$. microphylla Lindl. Une espèce s'est maintenue mais sa détermination n'a pa ètre précisée. 


\section{GenRe Sorbus}

Les deux espèces du genre Sorbus, 1'Alisier blanc (Sortus aria (L.) Grantz et le sorbier des oiseleurs (Sorbus aucuparia L..) appartiennent à la flore spontanée de l'Aigoual. Mais ils ont en outre été plantés. Le sorbier des oiseleurs, en particulier, a été planté en alignement le long des différents sentiers parcourant le secteur Europe, ainsi que sur la terrasse du chalet laboratoire. II a partout fort bien réussi.

\section{Genre Chaenomeles}

Un sujet de Chaenomeles japonica (Thunb.) Lindl., planté à l'automne 1905, s'est maintenu dans le placeau 10.

\section{Genre Pyrus}

Deux espèces, Pyrus betudarfolia Bunge et $P$. cathayensis Hunsley, plantées sans doute en 1907, sont portées sur la liste des espéces cultivées sans succès.

\section{Genre Rubus}

Rubus odoratus $\mathrm{L}$.

Espice américaine à grandes fleurs, introduite à l'automne 1905, s'est maintenue le long du ravin Thunberg (placeau 6).

\section{GENRE RosA}

Resa ragosa Thunb.

Fspice asiatique, planté par Flahaul à l'automne 1905; s'est maintente le long du ravin Thunberg.

\section{Genfe Prunus}

Prunus brigantiaca Vill.

Cette espèce, endémique dans les Alpes du Sud, a èté introduite d'abord en 1903, dans les placeaux affectés ultérieurement aux espèces asiatiques (plants recus de la région de Barcelonnette (B.A.), puis en 1909 (recus de M. Cuampsaur à Dignes (B.A.), Ce prunier s'est parfaitement maintenu ct est actuellement représenté par de nombreux pieds qui fructifient.

\section{Prunus laurocerasus L.}

74 sujets plantés à lautomne 1905, sont notés par FLAнAULt sur la liste des espèces cultivées sans succès satisfaisant (1910).

\section{Prumus padus $\mathrm{L}$.}

Un pied. planté en 1907. s'est maintenu jusquà ces dernières années dans le placeau 21 , quoique totalement dominé par la végétation environnante. 
Prunus serotina Ehrh.

Cette espèce de cerisier à grappes américaine est représentée par un sujet de $4 \mathrm{~m}$ de haut, assez vigoureux (placeau 22).

Prunus ancricana Marsh., $P$. orthosepala Koehne, $P$. maritima Marsh, $P$. cerasifera L. var. atropurpurea Jaeg ( $-P$. pissardi Carr.) sont mentionnés par FLAHAULT comme cultivés sans succès (1910).

\section{LEGUMINEUSES}

Les espèces suivantes ont été plantées sans succès: Amorpha fructicosa L., Gleditsia macracantha Desf., Wisteria sinensis, $W$. japonica Sieb. et Zucc., Cercis canadensis L.., Caragana brevispina Royle, Laburnum anagyroides Med. Seul, Robinia pseudoacacia L. s'est maintenu et est représenté par des sujets peu vigoureux. Enfin, Laburnum alpinum Bercht et Prsl. est représenté près du jardin alpin.

\section{EI.AEGNACEES}

\section{Genre Hippophae}

Hippophae rhamnoides L.

Cette espèce, fréquente dans les Alpes, s'est maintenue assez longtemps. NèGRe [10], RoL [13].

\section{Genke Elaneagnus}

Elacagnus umbellata Thunh.

Espèce introduite sans succès en 1907 (Flatuelr, 1910). 


\section{CONCLUSION}

L'Arboretum de l'Hort de Dies peut étre considéré comme un excellent exemple d'un type d'arboretum que l'on peut qualifier d'écologique. En effet, le site de l'Hort de Dieu, inclus dans un périmètre de restauration du massif montagneux de l'Aigoual. s'était révélé difficile à reboiser: d'une part, en raison de l'état de dégradation extrême de la végétation forestière naturelle, il n'était pas possible d'envisager la reconstitution, par simple protection. de la hêtraie montagnarde ou thermophile, forêt climatique, comme cela a été fait dans d'autres cantons voisins. Par ailleurs, différentes plantations effectuées avec des essences et des techniques qui avaient donné satisfaction dans d'autres stations du même massif, avaient jusque là à peu près totalement échoué.

II était donc nécessaire d'explorer les possibilités offertes jar les essences exotiques. Mais la première difficulté était d'effectuer un choix parmi ces essences dont le comportement était alors mal connu. Or, il faut tout d'abord que ces essences puissent supporter les conditions très rudes de la station: à une altitude moyenne de $1300 \mathrm{~m}$, sur des sols schisteux généralement rocheux. en climat méditerranéen montagnard, avec des précipitations annuelles certes très fortes mais irrégulièrement réparties et de fréquentes chutes de neige lourde, l'Hort de Dien est en outre, en raison de sa situation topographique, particulièrement exposé aux vents violents. De plus, ces essences doivent donner une production lignetse correspondant aux besoins de la conjoncture économique.

Pour étudier le comportement des essences exotiques dans de telles conditions, il fallait mettre en place une grande variété d'espèces : c'est ce qui a été fait à partir de 1903, d'abord par le Professeur Flahault, puis par la Station d'expériences et de recherches forestières de Nancy. Les renseignements recueillis au cours de cette expérimentation sur le comportement ainsi que sur l'intérèt que ces espèces présentent pour la production ligneuse, en quantité comme en qualité, permettent maintenant d'éclairer ce choix. Si les renseignements ne sont toutefois pas aussi précis que ceux qui auraient pu être obtenus par l'exploitation des résultats d'une plantation comparative installée suivant un dispositif permettant l'inter- 
prétation statistique, ils donnent néanmoins un prenier classement précieux des très nombreuses essences susceptibles d'ètre plantées. Ils permettent aussi précisément de choisir les quelques essences qui pourraient être retentues pour une expérience plus rigoureuse mais nécessairement limitée à un nombre restreint d'espèces, à mettre en place ultérieurement.

La longueur de la période d'observation, qui renferme des conditions climatologiques exceptionnelles telles que 1'hiver 1956, renforce la valeur de l'expérience, car les arbres ont eu à supporter effectivement une grande variété de saisons.

C'est certainement les résultats obtenu- avec les essences résineuses qui sont les plus intéressants: il faut préciser en premier lieu qu'à lexception du Pin sylvestre, spontané sur le versant atlantique du massif de l'Aigoual, tous les antres résineux doivent ètre considérés comme des exotiques, c'est-à-dire des essences étrangères à la flore spontancé, que ces essences appartiennent à la flore française (Abies alba, Picea abies, Pinus uncinata, Larix decidua) ou à des flores de régions beaucoup plus lointaines. Si les essences francaises précitées penvent être encore utilisées, les résultats des plantations expérimentales de l'Hort de Dieu montrent que d'autres essences exotiques peuvent être préférées pour les reboisements futurs.

Ainsi, dans le genre Abies, à côté du Sapin pectiné, d'autres espèces se sont révélées particulièrement intéressantes. Parmi les Sapins des régions circumméditerranéennes, c'est certainement Abics nordmanniana qui a donné les meilleurs résultats, avec une croissance au moins équivalente à celle du Sapin pectiné. Au contraire. dans ce même groupe géographique, l'utilisation d'Abies échlalonica est à proscrire à une altitude aussi élevée. malgré la vigueur de sa végétation, en raison des gélivures fréquentes. Ahies numidica et Abies pinsapo apnaraissent comme très nettement inférieurs, Parmi les sapins d'origine américaine, denx espèces méritent d'ètre considérées dès maintenant conme des essences de grande valeur pour les zones d'altitude élevéc dans les Cévennes méridionales: C'est d'ahord Abies procera (A. nobilis), quoiqu'on puise craindre des sécheresses estivales en sol superficiel, et Abies lonviana, dont les croissances sont très fortes. Il reste toutefois à préciser. en ce qui concerne Abies loaciana, dont la valeur spécifique est débattue puisqu'elle est souvent considérée comme une simple forme géographiaue d'Abies concolor, quelles seraient les meilleures origines, celle des arbres de l'arboretum n'étant pas connue. II en est de mème pour Abies concolor, dont les résultats à l'Hort de Dient ne sont pas nets. De nouvelles introductions d'origines précises sont à envisager. car une grande variabilité infraspécifique peut exister chez une essence dont l'aire spontanée est étendue et morcelée. I1 est regrettable qu'un autre Sapin. Abies arandis, qui a fait preuve d'une croissance si forte dans l'arboretum de La Foux, sur le ver- 
sant atlantique de 1'Aigoual et à plus faible altitude, ne soit pas représenté également à l'Hort de Dieu par une plantation ágée.

Les autres Sapins, américains (. Abics lasiocarpa, A. balsanca) ou asiatiques $(A$, veitchii) apparaissent très inférieurs.

Le Sapin de Douglas (Pscudotsuga mensicsii var. mensiesii) se montre très vigoureux et se réensemence abondamment mais, à une altitude aussi élevée, il apparait que son emploi doit être réservé aux sols profonds et surtout aux stations les plus abritées des vents.

Parmi les épicéas, aucum ne se montre supérieure à Picca abies. En effet, Picra omorica et $P$. oricutalis quoique parfaitement adaptés aux conditions climatiques, ont une croissance très nettement inférieure. Piça hondocnsis, très branchu, ne présente pas d'intérêt forestier. Picca pungcns est peu longévif et. seul Picea cngelmanni, dont l'introduction est encore récente, mérite d'être suivi et, sil $y$ a lieu, réintroduit.

Les résultats obtenus à l'Hort de Dieu confirment la grande plasticité du Mélèze du Japon (Larix leptolepis) qui s'accommode fort bien des conditions rudes des climats montagnards.

L.es Cẻdres doivent être, ici, pratiquement éliminés comme essences de reboisement. Le Cèdre de 1'Himalaya (Cedrus deodar) a déçu dès les premières années. Cenx du Liban $(C$. libani) et de I'Atlas (C. atlantica) n'ont pas survécu à l'hiver 1956. L'expérience de l'Hort de Dieu permet de fixer à $900 \mathrm{~m}$ environ, dans le massif de I'Aigoual, l'altitule pratique d'utilisation du Cèdre de l'Atlas, essence couramment utilisée en reboisement dans les montagnes de la région méditerranéenne française.

Les Pins, à l'exception des espèces dont les stations spontanées sont précisément les étages montagnards ou subalpins, ont mal résisté aux conditions dures de l'Hort de Dieu. C'est ainsi que les grandes espèces de reboisement telles que le Pin sylvestre. le Pin noir d'Autriche, le Pin laricio de Corse, ont donné des résultats movens ou mécliocres, L'échec des pins d’origine américaine ( $P$. strolus, $P$, rigida, $P$. ponderosa, $P$. banksiana) est à peu près complet. Seuls les Pins montagnards tels que Pinus combra, $P$. peuce, $P$. leucodermis, $P$. uncinata et $P$. pumilio sont vigoureux mais ont tous une croissance lente.

Ouelques fetrillus ont été également introduits avec succès. C'est le cas de l'Aune blane (Alnus incana) qui a prospéré le long des différents ravins, des bouleaux qui ont constitué des peuplements pouvant ètre maintenant utilisés comme abris, de l'Erable sycomore et du Frêne dans les stations fraiches, mais le Charme, le Coudrier, les Ormes, les Sorbiers ne peuvent constituer des éléments de reboisement intéressants que dans la mesure où il apparait nécessaire de créer des peuplements mélangés.

Du point de vue phytogéographique, les expériences de 1'Hort de Dieu sont également intéressantes. Elles montrent en effet que 
la valeur, en matière d'introduction d'espèces, des différentes régions florales est inégale. Ainsi, en ce qui concerne l'Amérique du Nord, il est certain que c'est la région Ouest qui peut nous fournir les espèces forestieres de beaucoup les plus intéressantes pour les reboiseurs français, telles que Pseudotsuga menzicsii, Abies procera, $A$. lowiana et peut ètre $A$. concolor. Au contraire, les essences de la région florale Est, aussi bien résineuses que fetuillues, ne donnent dans l'ensemble que des résultats très moyens.

Toutes les espéces montagnardes de la région florale d'Europe et du Moyen-Orient, notamment du genre Picea (Picea abies, P. omorica, $P$. orientalis), du genre Abies (Abies alba, $A$, nordmanniana, A. cephalonica) et du genre Pinus (Pinus cembra, P. peuce, $P$. leucodermis, $P$. uncinafa) ont toutes montré de bons caractères d'adaptation, même si leur intérêt en tant qu'espèce de production ligneuse est divers.

Enfin, toutes les espèces originaires d'Extrème-Orient n'ont donné que des résultats médiocres, à l'exception de Larix leptolepis, mais les possibilités de la flore asiatique, notamment de la flore montagnarde, sont loin d'avoir été totalement explorées.

Décembre 1963. 


\section{Index alphabétique des noms des espèces mentionnées au Catalogue}

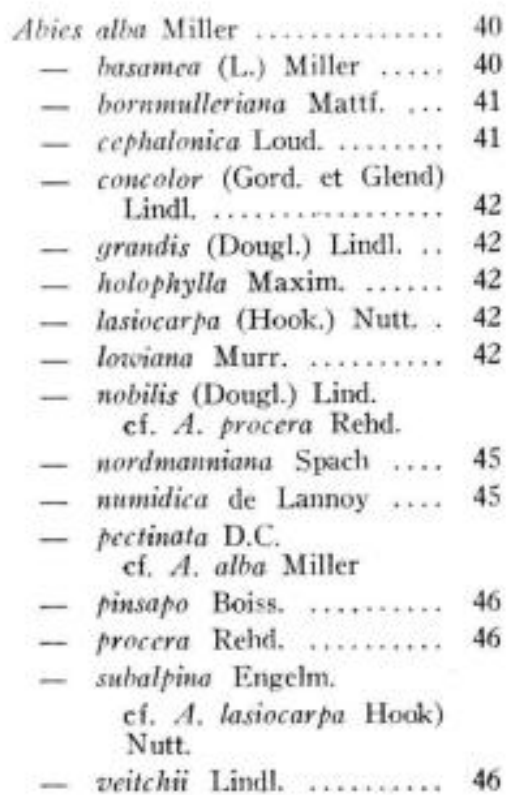

Abelia uniflora R. Drown. ..... 63

Acer campestre L. ............ 62

- insigne Boiss. ........... 62

- macrophyllum Pursh. .... 62

- negundo L.............. 62

- pensylvanicum L. ........ 62

- platanoides L. ........... 62

- pseudoplatanus L. ........ 62

- saccharinum L. ......... 62

Aesculus californica (Spach)

Nutt. ............. 62

- $\times$ carnea Hayne .... 62

- hippocastanum L. .... 62
- macrostachya Michx cf. A. parviflora Walt.

- parziflora Walt. ..... 62

- rubicunda Loisel

cf. A. $\times$ carnea

Hayne

Ailanthus altissima (Mill) Swingle $\ldots \ldots \ldots \ldots \ldots \ldots, 61$

- glandulosa Desf. ef. A. altissima Mill.

Alnus incana (L.) Moench .... 57

- viridis D. C. ........... 58

Amorpha fraticosa L. ....... 68

Araucaria arancana K. Koch. ., 56

- imbricata Pav. cf. A. arancana $\mathrm{K}$. Koch.

Arole

cg. Pinus combra L.

Aucuba japonica Thunb.

Aune blanc

of. Alnus incana (L.) Moench.

Aune vert

cf. Alnus viridis D. C.

Berberis amurensis Rupr. ,.,., 65

Betula alleghanicusis Britton. .. 58

- Ienta L. .............. 58

- Iutea Michx.

cf. B. alleghanicnsis Britton.

- pubescens Ehrh. ....... 58

- verrucosa Ehrh. ........ 58 
Biota orientalis Endl. ......... 56

Broussonetia papyrifara (L.) Vent ...............6 61

Calocedrus decurrens (Torrey)

Florin ............. 56

Caragana brevispina Royle

Carpinus americana Michx. ef. C. caroliniana Walt.

- betulus L. ............ 59

- caroliniana Walt. ...... 50

Carya illoneensis Wangenh. .... 61

- olizaeformis Michx. cf. C. illoncensis Wangenh

Cedrus atlantica Manetti ...... 50

Cèdre de l'Atlas ci. C. atlantica Manetti.

- deodora (Roxb.) Loud. .. 51

Cèdre du Lihan ef. Cedrus libani Losd.

- libani Loud. 51

Celtis caucasica Willd.

- occidentalis L. .......... 60

- sinensis Pers. ........... 60

Cercis canadensis L........... 68

Chaenomeles japonica (Thunb.) Lindl.

Chamaecyparis lanusoniana (A Murr.) Parl. ........... 57

Charme

ef. Carpinus betulus.

Chène pédonculé cf. Ouercus robur L.

Chène sessile cf. Ouercus petraca Liebl.

Cornus stolonifera Michx. ...... 63

Corylus avellana L. ............ 59 - colurna L............ 59

Cotinus coggygria Scop. ..... 61
Cotoncaster microphylla Lind. . 66 - multiflora Bge.... 66 - reflera Carr. cf. multiflora Bge.

Cotdrier

ef. Corylus avellana $\mathrm{L}$.

Cyprès chauve

cf. Taxodium distichum L.

Destsia silholdiana $\mathrm{Max} \ldots . . .66$

- staminca $\mathrm{R} . \mathrm{Br} . \ldots \ldots .66$

Diervillea florida Sich. et Zucc. ci. Weigelia florida Sieb, et Zucc.

Elcagnus umbellata Thunb. .... 68 Êpicéa cf. Picea abies (L.) Karts.

Erable plane cf. Acer Natanoides 1.

Erable sycomore cf. Acer pseudoplatanus L.

Evonymus latifolius Scop. ..... 63

Fagus americana L. cf. F. grandifolia Fhrh.

- grandifolia Ehrh. ....... 58

Forsythin suspense Vaht. ...... 64

- viridissima Lindl. .. 64

Fraxinus alba Marsh. .......... 64

- americana L. ........ 64

- excelsior L. ......... 64

- lanceolata Borkh.

cf. F. pensylaraica Marsh.

- mandsharica Rupr. .. 64

- oregona Nutt. ...... 64

- pensylaanica Marsh. .. 64

- pubescens Lam. ...... 64

Frène

ef. Fraxinus excelsior.

Gyukyo biloba

Gleditsia macracantha Desf. ... 68 
Heyderia decurrens Torr. Roch. ci. Calocedrus decurrens Torr, Florin.

Hippophae rhannoides 1. ...... 68

Holodiscus discolor (Pursh)

Maxim

Mcx aqquifolium L. ............. 54

Jasmimum Lindl. ............. 64

Juglans cincrea 1. ............., 61

- nigra 1. ............. 61

Juniperus sabrina L. ............ 57

- virginiano L. ....... 57

Laburnum alpinum Bercht et Prsl. ......... 68

- anagyroides Med. ... 68

Larix decidua Mill. ........... 50

- ruropea D. C cf. L, decidua Mill

- leptolepis (Sieb. et Zucc.) Gord.

Libocedrus decurrens Torr.

cf. Calocedrus decurrens (Torr.) Florin.

Ligastrina pekinensis Regel

ef. Syringa pekinensis Rupr.

Liqustrum amurense Carr. ..... 65

- compactum Hook et Thomas ........ 65

- ibota Sicb. et Zuce. 65

- quihoui Carr. ...... 65

- sinense L. var. stamitonii (D. C.) Rehd. 65

- yиялаnense L. Henry 65

Liquidambar styraciflua L. .... 66

Lonicera alpigena L........... 63

- brachypoda D.C. cf. L. Japonica Thunb.

- iberica Bieb. var. microphylla Dip. ..... 63

- japonica Thunb. ..... 63
- kesselringii Reg.

cf. L. orientalis L.am.

- ledebourri Eschsch. .. 63

- orientalis Lam. ...... 63

- prolifera Rehd. ...... 63

- pyrenaica L. ........ 63

- sempervirens L. ..... 63

- sullizuntii Gray

cf. L. prolifera Rehd.

Mahonia aquifolium (Pursh)

Nutt.

Mélèze d'Europe

ci. Larix decidua Mill.

Méléze du Japon

cf. Iarix leptolepis

(Sieb, et Zuce.) Gord.

Noisetier

ef. Corylus avellana L.

Orme de montagne

ef. Ulmus glabra Huds.

Oslrya virginiana (Mill.)

K, Koch. ............ 59

Parrotia persica C.A. Mey...... 66

Parrotiopsis jacquemontiana ... 66

Philadelphus ............... 66

Physocarpus opulifolius (L.)

Maxim. ............... 66

Picea abies (L.) Karst. ........ 47

- ajanensis cf. P. hondoensis

- alcokiana Carr. ......... 48

- asperata Mast ......... 48

- engelmani Parry ...... 48

- excelsa Link.

cf. P. abies (L.) Karst.

- hondoensis Mayr. ...... 48

- morinda Link.

cf. P. smithiana Noiss.

- omorica (Pancic) Purkine. 49

- orientalis (L.) Link. .... 49

- pungens Engelmann ..... 49 
- sargenfiana Rehd. et

Wils. .............. 49

- smithiana Boiss, ....... 49

Pin àt crochets

cf. Pinus uncinata Ramond.

Pin cembro

cf. Pinss cembra I.

Pin de Banks cf. Pinas banksiana Lamb.

Pin de l'Himalaya

cf. Pinus griffithii MC

Clelland.

Pin gris

cf. Pinus banksiana Iamb.

Pin laricio

cf. Pinss nigra ssp. laricio.

Pin rampant. cf, Pinus mughas Scop.

Pin Weymouth ef. Pinus strobus.

Pinus banksiana Lamb. ....... 51

- cembra I. ............. 51

- contorta Douglas ........ 52

- excelsa Wall. cf. P. griffirhii

MC Clelland....... 52

- leucodermis (Ant.) Markgraf ................ 52

- mughus Scop. ........, 52

- nigra Arnold ssp. laricio

Poiret var, calabrica .. 53

- nigra Arnold ssp. laricio

var. corsicana ....... 5,3

- nigra Arnold ssp. nigricaus var, austriaca .... 53

- peace Grisb. ........... 53

- ponderosa Laws. ........ 53

- pumilio Haenke ......... 54

- resinosa Ait. ........... 54

- rigida Mill. ............ 54

- strobus 1. .............. 54

- silvestris I. .......... 54

- uncinata Ramond. ...... 55

Polygonum sacchalinense

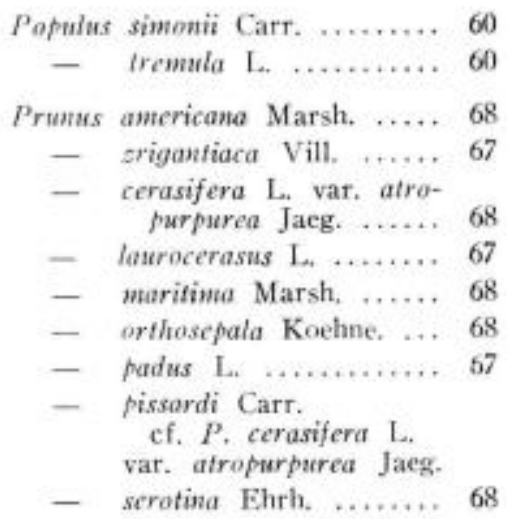

Psemdolarix amabilis (Nels.)

Rehd. ............. 50

Pseudotsuga douglasii cf. $P$. mensiesii.

- mensiesii (Mirb.) Franco. ........ 47

Pferocarya stenoptera D. C. ... 61

Purus betulacfolia Bunge ...... 67

- cathayensis Hunsley .... 67

Ouercus ilicifolia Wangenh. .... 58

- macrocarpa Michx. .... 58

- pedunculata Ehrh. cf. Q. robur L.

- petrata Liebl. ......... 59

- robur L.............. 59

- rubra L. ............ 59

Rhamnus alpina L. .......... 64

- frangula L. ......... 64

- pallasii Fish. et Mey .. 64

Rhododendron ferruginemem L.. .. 65 $\begin{array}{lllll}\text { - } & \text { hirsutum } & \text { L. } & \ldots . & 65 \\ \text { - } & \text { ponticum } & \text { L. } & \ldots . & 65 \\ \text { - } & \text { smirnovii } & \text { Trautv. } & 65\end{array}$

Khus cotimas I. cf. Cotinus coggygria Scop.

Robinia psesdoacacia L. ...... 68

Rosa rugosa Thunb. ......... 67

60 Rubus odoratus L. .......... 67 
Salir caesia Vill.

- capraca L.

- grandifolia $Q$.

- purpurea L.

- pyrenaica Gouan

Sapin de Douglas

cf. Pseudotsuga mensiesii (Mirb.) Franco.

Sapin pectiné cf. Abies alha Miller.

Sequoiadendron giganteum (Lindi) Buchholz ....... 56

Sequoia sempertirens (D.Don) Fndl. ............. 56

Sorbus aria (L.) Grantz ..... 67

- ancuparia L. ......... 67

Spireac salicifolia L. ......... 66

Symphoricarpos albus Blake .. 63

Syringa pekinensis Rupr. . . . . 65

- persica L. ........... 65

- vulgaris L. .........65 65

Taxodium distichum (L..) , . , 56

Tarus baccata L. .......... 57

Thuja occidentalis L. ........ 57

- plicata Donn. ........ 57

Thujopsis dolobrata Sieb, et Zuce. 57
Tilia grandifolia Ehrh. cf. T. platyphyllos.

- platyphyllos Scop. 65

Tsuga hookeriana (A. Murr)

Carr. cf. T. mertensiana (Bong) Carr.

- mertensiana (Bong Carr.) 50

Ulmus americana L. ......... 60

- glabra Huds. ......... 60

- montana Witer

cf. U. glabra Huds.

- scabra Muller cf. $U$. glabra Huds.

Yiburnum lantana L. ........ 63 - opulas L. ........ 63

- pubescens Pursh. .. 63

- venosum Brit. cf. $V$. pubescens Pursh.

Wcigclia florida (Sieb. et Zucc.)

A. DC. .............. 63

Wisteria japonica Sieb et Zuce. 68 - sinensis DC. ....... 68

Zanthorylum americanum Mill. 61 piperetwm (L.)
DC.............. 61

Zelkova acuminata Planch. cf. Z. serrata Thunb. serrata (Thunb,) Mak, 61 


\section{BIBI.IOGRAPHIE}

(1) Braun-Blanguet. - Flore du Mascif de l'Aigoual. Mémoires de la Sté d'Etude des Sciences Naturelles de Nimes, $\mathrm{n}^{*} 4,1933$.

(2) Debazac (E.-F,), - L'arboretum d'Amance. Annales de l'Ecole Nationale des Eautu et Forêts et de la Station de Recherches et Expériences, t. XVIII, fasc. 3, 1961.

(3) Debazac (E.-F.). - L'arboretum de La Foux (massif de l'Aigoual). Retue Forestiche Française, nov. 1962, p. 909-918.

(4) Eмывксек (L..), - Traité de Botaniøue. Les Végétaux vasculaires. Paris, 1960 .

(5) Flahaclit (Ch.). - Rapport présenté au conseil de l'Université au sujet des jardins botaniques de l'Aigoual. Montpellier, 19 p., 1904.

(6) Finault (Ch.). - Georges Fabke. Bulletin Société Etudes at Sciences naturelles de Nimes, vol. XI., 1912-13, 20 p.

(7) Fl.augèkrs (A.), - Plantes exotiques et arboretums dans les Céventies méridionales. Retue des Eaur et Foríts, 65, p. 380-388 et 449-460, 1929.

(8) Galzin (J.). - Les forèts de l'Aigoual. Revue économigue de la Chambre de Commerce de Nimes - Ua's - le Vigan, p. 21-37, 1953.

(9) Guinier ( $\mathrm{Ph}$.$) . - Charles Flahault (1852-1935). Reviee des Ean.r et$ Forêts, 73 , p. $397-411,1935$.

(10) Nègre (M.). - Les reboisements du massif de l'Aigoual. Mémoires de la Societe d'Etude des Sciences naturelles de Nimes, $n^{\circ} 3,135$ p., 1931.

(11) Nozeran (R.), Roux (J.) et Valdeyrox (G.). - L'évolution depuis son sbandon (1914) des jardins botaniques créés par Ch. Flahault dans le massif de l'Aigoual. I. L'Hort de Dien, plantes herbacées et arbustes du secteur Europe. Nafuralia Monspeliensia. 11, 1959.

(12) PARDÉ (J.). - Excursions dendrologiques dans la région de Nimes et de Montpellier. Bulletin de la Socifte dendrologizue de France. $\mathrm{n}^{\circ} 12$, p. $119-132,1909$.

(13) RoL (R.). - Les arboretums du massif de l'Aigoual. Rapport au congrès international des Stations de Recherches forestières, Sopron, 1936.

(14) Rot. (R.). - Le massif de l'Aigoual. Etude botanique et forestière. Bulletin de la Sociéte botanique de France, $80^{\circ}$ session extraordinaire, 100 , p. $39-46,1953$, 


\section{RÉSUMÉ}

L'Arboretum de l'Hort de Dieu fait partie d'un réseau de places expérimentales installées dans le Massif de l'Aigoual dans les Cévennes méridionales pour l'étude du comportement des essences exotiques.

Les premières plantations ont été faites en 1903 par le Professeur FLAHaut. Après 1928, cet arboretum a été géré par la Station de Recherches et Expériences forestières de Nancy.

Les plantation se trouvent entre 1250 et $1300 \mathrm{~m}$ d'altitude à $600 \mathrm{~m}$ au sud du sommet de l'Aigoual. Le sol est constitué par des schistes. Le climat est du type montagnard avec des influences méditerranéennes marquées. La hauteur moyenne des précipitations annuelles est de $2000 \mathrm{~mm}$ environ avec un maximum d'automne très marqué et un minimum d'été. La neige est abondante et le brouillard fréquent. Les vents sont extrèmement violents avec une dominance des directions $\mathrm{N}$ et NW.

Au moment des plantations, le site était à peu près totalement déboisé. Le groupement forestier climacique est une hêtraie montagnarde à moder mais, aux expositions les plus chaudes, cette hếtraie a un faciès thermophile avec chêne pubescent.

Les conditions écologiques très dures expliquent les échecs des essais de reboisement effectués avant 1903 dans cette station alors que d'importants travaux de reforestation avaient été réalisés avec succès dans l'ensemble du Massif de l'Aigoual avec des essences hahituelles: Mélèze đ'Europe, Epicéa, Pin à crochets et Sapin. Aussi, le but de cette expérimentation était d'étudier le comportement de nombreuses essences susceptibles d'ètre utilisées.

Les plus anciennes introductions ont actuellement entre 50 et 60 ans. Il est done possible maintenant de tirer des enseignements précieux sur l'intérêt forestier pour le reboisement des nombreuses essences essayées.

Parmi les Sapins du groupe circumméditerranéen, c'est Abics nordmanniana qui a donné les meilleurs résultats. Abies numidica et A. pinsapo apparaissent très nettement inférieurs. Parmi les Sapins américains, c'est Abies procera et $A$. lowiana qui ont les crois- 
sances les plus fortes. Abies concolor n'a pas donné des résultats nets. Abies lasiocarpa et $A$. balsanca sont beaucoup moins intéressants.

Le Sapin de Douglas (Pseudotsuga menzicsii) est très vigoureux mais se montre très sensible aux vents. Aucun des épicéas essayés (Picea omorica, $P$. orientalis, $P$. hondocnsis, $P$. pungens, $P$. cngelmanni) n'est supérieur à Picca abies. L'Épicéa de Sitka n’a pas été introduit.

L.es Cèdres de l'Atlas et du Lilkan ont été éliminés par les basses températures exceptionnelles de l'hiver 1956.

Parmi les Pins, seuls les pins typiquement montagnards tels que Pinus cembra, $P$. peuce, $P$. leucodermis, $P$. uncinata, $P$. pumilio se montrent vigoureux mais ont des croissances faibles. Beaucoup d'échecs ont été enregistrés dans l'introduction des espèces feuillues, à l'exception des espèces montagnardes dont l'intérét au point de vue de la production, est faible. 


\section{SUMMARY}

The Arboretum of the Hort de Dieu belongs to the network of experimental plots set up in the Aigoual mountain range of the southern Cevennes in order to study the behaviour of exotic species.

The earlier plantations were made by Professor Flahaut in 1903. After 1928, this arboretum has been managed by the Forest Experiment Station at Nancy.

The plantations are located between 1250 and $1350 \mathrm{~m}$. in altitude, $600 \mathrm{~m}$. south of the Aigoual highest point. The ground is composed of schists. The climate is of the mountain type with market mediterranean influences. The mean height of annual rainfall is around $2000 \mathrm{~m}$. with a strongly marked maximum in autum and a minimum in summer. There are lots of snow and fog is frequent. Winds are particularly strong with a dominance in the northward and nord-westward directions.

When the plantations have been set up, the site was almost entirely treeless. Its climacic forest association is a mountain beech forest with moder, but in warmer exposures this beech forest assumes a termophilots aspect with pubescent oak.

The severity of ecological conditions explains the failure of the reforestation trials made in this station before 1903 whereas extensive regorestation with usual species, European Larch, Spruce, Mountain Pine ( $P$. uncinata) and Fir, had been successfully carried out in the whole Aigotual mountain range. The purpose of this experimentation was thus to study the behaviour of numerous species liable to be used for reforestation.

The earlier introductions are at present hetween 50 and 60 years old. It is therefore possible now to derive valuable information on the suitability for reforestation of the numerous species tried.

Among firs of the circummediterranean group, Abies nordmanniana was the most successful. Abies numidica and $A$. pinsapo appear much less satisfactory. Among american firs, Abies procera 
and A, lowiana showed the largest increments. Abies concolor did not give significant results. Abies lasiocarpa and $A$. balsamea were much less interesting.

Douglas Fir (Pseudotsuga menzicsii) is very strong but very susceptible to winds. None of the spruces tried (Picea omorica, $P$. orientalis, $P$. hondocnsis, $P$. pungens, $P$. engelnanni) has given better results than Picca abies. Sitka Spruce has not been introduced.

Cedars from the Atlas and Lebanon have been eliminated by untusually low temperatures during the winter of 1956.

Among pines, only typical mountain pines such as Pinus combra, $P$. peuce, $P$. leucodermis, $P$. uncinata, $P$. pumilio appear strong. but grow rather slowly. Many failures have been recorded in the introduction of hardwood species, except for mountain species of little interest in regard to forest production. 


\section{ZUSAMMENFASSUNG}

Das Arboretum von "Hort de Diet $n$ ist Teil eines Netzes von Versuchsflächen die im Aigoualgebirge, in den südlichen Cevennen, eingestellt wurden. Der Zweck dafür war das Erforschen der Lebensbedingungen exotischer Holzarten.

Die ersten Anpflanzungen wurden durch H. Professor FLAHAUT in Jahre 1903 durchgeführt. Nach 1928 wurde dieses Arboretum durch die "Station de Recherches et Expériences forestières " von Nancy bewirtschaftet.

Die Pflanzungen befinden sich zwischen einem Höhestreifen von 1250 und $1350 \mathrm{M}$, und $600 \mathrm{M}$ südlich des Gipfels von Aigoual, Der Grundboden besteht aus Schiefergestein. Das Klima ist von gebirgisches Typus mit stark fühlbaren mittelländischen Einflüssen. Die Mittelhöhe der jährlichen Niederschlägen erreicht $2.000 \mathrm{MM}$, mit sehr fühlbarem Maximaherbst und Minimasommer. Sehr reicher Schnee und oft Nebelbildung. Die Windstösse, sehr heftig und gewaltig, heziehen eine Hauptrichtung $\mathrm{N}$ und NW.

Zur Zeit der Anpflanzungen war die Landschaft fast völlig entholzt. Die klimazische Waldgesellschaft ist ein Bergbuchenwald, über Moderboden, erhält aber in den heissesten Aussetzungen einen thermophilen Fazies mit Flaumeichen.

Der arg strenge ökologische Zustand gibt Grund an über die misslungene, vor 1903 in diesem Standort ausgeführten Aufforstungsversuche, alulann beträchtliche Waldanbauarbeiten im gesamten Aigoualgebirge insgesamt mit Erfolg gekrönt wurden, dies mit gewohnten Holzarten : Europalärche, Fichte, Bergkiefer $(P, u n$ (inata) und Tanne. Das Ziel also dieses Versuches war das Studium des Verhaltens der vielen hier zur Anwendung kommenden Holzarten.

Die ältesten Einführungen zählen jetzt einen Alter von 50 und 60 Jahren, Es ist daher jetzt möglich kostbare Belehrungen über das Anbatinteresse der vielen zum Versuch eingeführten Holzarten.

Unter den Tannen der Zirkummittelländischen Gruppe, ist Abies nordmanniana die erfolgreichste. Abies numidica und $A$. pinsapo scheinen ihr schraff unterlegen zu sein. Unter den amerikanischen Tannen, sind es Abies procera und A. lowiana die die stärksten 
Wuchsleistungen besitzen, Abies concolor hat keine klare Resultate gegeben. Abies lasiocarpa und A. balsanea bieten viel weniger Interesse.

Die Duglasie (Pseudotsuga menziesii) ist sehr kräftig aber den Windstössen entgegen sehr empfindlich. Keine der zur Probe angebauten Fichten (Picea omorica, P. orientalis, $P$. hondoensis, $P$. pungens, $P$. engelmanni) ist der Picea abies überlegen. Die Sitkafichte wurde nicht eingeführt.

Die Atlas und Libanonzeder wurden durch die atusserordentlich niederen Temperaturen des Winters von 1956 ausgemerzt.

Unter den Kiefern, allein nur die typischen Bergkiefern so wie Pinus cembra, $P$. peuce, $P$. leucodermis, $P$. uncinata, $P$. pumilio beweisen sich kräftig, haben aber schwache Wuchsleistung. Das Einpflanzen der Laubhölzer führte zu vielen Misserfolgen, dies aber mit Ausnahme der Bergsorten deren Forstinteresse, betreffs der Holzproduktion nur wenig Wert aufweist. 